\title{
Indagini quantitative negli studi delle disabilità e dei DSA: problemi e prospettive in ambito nazionale e internazionale
}

\section{Lucia Chiappetta Cajola}

Università degli Studi «Roma Tre», Dipartimento di Scienze della Formazione

doi: 10.7358/ecps-2014-009-chia_lucia.chiappettacajola@uniroma3.it

\section{QUANTITATIVE INVESTIGATIONS IN STUDIES \\ ON DISABILITIES AND SPECIFIC LEARNING DISORDERS: \\ NATIONAL AND INTERNATIONAL PROBLEMS AND PROSPECTS}

\section{Abstract}

Gathering, organizing, describing and interpreting data are the steps which, in particular, characterize the quantitative investigation process. They are considered to have growing importance in a society where it is fundamental to fully comprehend the great and complex changes underway, in order to be able to effectively respond to the challenge of improving the quality of education for all students. Starting from this premise, this contribution aims to provide a picture of the quantitative investigations carried out at both national and international level within studies of disabilities and of specific learning disorders, and highlights the critical aspects linked to the many different survey tools and methods employed for obtaining data and information in various countries. This plethora of methods and tools does not make for having statistically comparable data to describe the problems hindering student learning and participation in school life, and to obtain the various trends. The article maintains that the efforts to empirically and operationally account for such a complex reality makes it necessary to enhance actions for integrating competencies with regard to the renewed and decisive support and also involvement of authoritative national and international bodies which can methodologically reorient investigation procedures by overcoming the critical aspects linked to the use of concepts, definitions and classifications as well as the criteria for data-gathering and database management, also bearing in mind the many actors involved and the relative legislative and normative constraints. 
Keywords: Disabilities, Evidence based education, International classification of functioning, Quantitative investigations, Specific learning disorders.

\section{INTRODUZIONE}

In una realtà sociale e culturale caratterizzata da un'attenzione crescente verso la molteplicità dei fenomeni in ambito educativo e scolastico, e delle rapide e complesse trasformazioni che contribuiscono a determinarli, è palese la necessità di disporre di informazioni sui bisogni formativi individuali e sul contesto di appartenenza, sia per assumere decisioni atte a promuovere, in un'ottica inclusiva, il successo formativo di tutti gli allievi, sia per accogliere la sfida del miglioramento della qualità dell'istruzione, irrinunciabile a livello nazionale e internazionale. Come è noto, nella prospettiva delle indagini quantitative, la validità dell'utilizzo di differenti strumenti e metodi scelti per la rilevazione delle informazioni e l'elaborazione dei dati dipende dalla capacità di questi di apportare significati statisticamente utili a descrivere $\mathrm{i}$ problemi che ostacolano l'apprendimento e la partecipazione degli allievi alla vita scolastica, e a fornire linee di tendenza sul loro andamento.

Tuttavia nelle indagini quantitative condotte specificamente nell'ambito degli studi delle disabilità e dei Disturbi Specifici di Apprendimento (DSA), la raccolta di dati statistici omogenei e confrontabili evidenzia alcune difficoltà prevalentemente ascrivibili a due principali criticità. La prima è connessa, per un verso, alle pluridefinizioni di disabilità determinate sia dalla complessità e multifattorialità che ne caratterizzano il concetto e il significato, sia dal modello teorico assunto (medico; sociale; biopsicosociale; delle risorse; dei diritti umani) e dalla qualificazione dei vari livelli di gravità; per l'altro verso, è correlata talvolta ad incertezze eziologiche e diagnostiche dei DSA a causa dell'utilizzo di diversi strumenti di accertamento, di eventuali comorbilità con altri disturbi, nonché di limitate esplorazioni epidemiologiche condotte al riguardo.

La seconda criticità riguarda, invece, aspetti propri delle modalità di registrazione delle informazioni nelle banche dati (Ostillio, 2005) ed altri specifici della metodologia della ricerca, quali gli obiettivi delle singole indagini, il campionamento, gli strumenti di misurazione adottati, le dimensioni temporali di riferimento, la stesura dei questionari con la relativa formulazione dei quesiti e le categorie di risposta adottate (Zammuner, 2000; Trinchero, 2002; Corbetta, 2003; Cannavò \& Frudà, 2012; Chiaro, 2012; Ciucci, 2012; Lucisano \& Salerni, 2012).

In questo quadro fortemente differenziato e frammentario entro cui è latente il rischio di discriminazione nell'offerta quantitativa, ma anche qua- 
litativa, di dati e statistiche sulla condizione di vita delle persone con disabilità e con DSA, in questo caso riferite all'ambito scolastico, appare dunque evidente che l'impegno di rappresentare sul piano empirico e operativo una realtà così complessa comporta la necessità sia di potenziare lo sforzo di integrazione di competenze, sia di sviluppare la consapevolezza dell'urgenza di un rinnovato e decisivo supporto e coinvolgimento di autorevoli organismi istituzionali, nazionali (MIUR, 2007-2009, 2010-2012; MIUR e Ministero della Salute 2012; ISTAT, 2013; Ministero degli Affari Esteri, 2013; Ministero del Lavoro e delle Politiche Sociali, 2013) e internazionali (HMSO-Warnock, 1978; UNESCO, 1997, 2012; WHO, 2001, 2007, 2011; OECD, 2005, 2012a, 2012b; UNICEF, 2013), in grado di ri-orientare metodologicamente le procedure di indagine, superando innanzitutto le criticità legate sia all'uso di concetti, definizioni e classificazioni, sia ai criteri di raccolta dei dati e di gestione dei relativi data-base, tenendo anche in conto la molteplicità degli attori coinvolti, nonché i vincoli di natura legislativa e normativa connessi.

Affinché le informazioni sulla disabilità e sui DSA possano infatti assumere una valenza statistica in ragione di fonti affidabili, l'esigenza di fondo è in definitiva quella di una loro messa a sistema, con l'obiettivo di progettare e disporre di nuovi ed efficaci flussi informativi (Domenici, 2006 e 2009) in grado di permettere una conoscenza approfondita di carattere quantitativo e, quando possibile, di carattere qualitativo, sulle condizioni di vita delle persone con disabilità e con DSA a livello locale e globale, supportando nello stesso tempo l'azione di monitoraggio del processo di inclusione scolastica, sociale e produttiva.

Il raggiungimento di tale obiettivo può infatti dar luogo ad una maggiore incisività degli sforzi in atto e delle azioni delle diverse istituzioni coinvolte, nei vari Paesi, nell'elaborazione dei documenti relativi alle indagini effettuate e dei successivi orientamenti programmatici negli ambiti dell'educazione e della formazione, vitali per l'esercizio dei diritti di cittadinanza, oltre che per l'attuazione delle politiche inclusive, indispensabili in una società civile.

È tuttavia da puntualizzare che, a fronte di una produzione, per certi versi ampia, di studi ed indagini quantitative per la disponibilità di dati ed informazioni in tali ambiti, condotti a differenti gradi di profondità riguardo al livello territoriale e nel merito degli aspetti affrontati, permane ancora irrisolto il problema di poter comparare, in particolare a livello internazionale, statistiche ed indagini quantitative sul tema della disabilità e dei DSA, e di conseguenza ancora lontano il superamento di tale difficoltà, a scapito della realizzazione di sistematici processi inclusivi e della possibilità di intraprendere azioni efficaci a tale scopo.

Ciò accade, oltre che da quanto evidenziato in precedenza, anche nel caso vengano impiegate metodologie di rilevazione rigorose e scientifiche 
(Lazarsfeld, 1966; De Landsheere, 1973; Kish, 1987; Campbell \& Stanley, 1996; Jenkins, 2000; Trinchero, 2002; Corbetta, 2003; Lumbelli, 1984, 2006; Domenici, 2006; Newbold \& Carlson, 2010; Hattie \& Gan, 2011; Hattie, 2012; Lucisano \& Salerni, 2012; McMillan \& Schumacher, 2013).

Di tali elementi problematici, dello stato dell'arte ad essi relativi e delle prospettive emergenti, si intende discutere nelle pagine seguenti del presente contributo, stante il dibattito internazionale aperto e quello nazionale riacceso da recenti interventi sia del legislatore in materia di DSA (Legge n. 170/2010) sia del MIUR $(2011,2012,2013 \mathrm{a}, 2013 \mathrm{e})$ in riferimento ai Bisogni Educativi Speciali (BES) degli alunni.

\section{INDAGINI QUANTITATIVE E COMPARABILITÀ DEI DATI: L'IMPEGNO DELLE ORGANIZZAZIONI INTERNAZIONALI}

Il problema appena delineato rientra nel quadro più generale di una politica culturale, evidentemente ancora da costruire, in grado di promuovere iniziative a carattere scientifico che possano costituire le basi per studi e ricerche dai quali rilevare informazioni comparabili ed universalmente interpretabili, come nel caso dei disturbi specifici di apprendimento e delle disabilità.

Tale necessità emerge anche dagli studi propri dell'educazione comparata (Bray, Adamson, \& Mason, 2007), nell'ambito della quale vengono condotte analisi in profondità che evidenziano in modo netto alcune problematicità sottese sia all'assunzione dei modelli teorici che alle scelte metodologiche nelle ricerche condotte in campo educativo. Una delle maggiori problematicità è relativa all'oggetto della comparazione che riguarda, nello specifico, gli approcci quantitativi e qualitativi, e ne evidenzia i punti di forza e di debolezza attraverso il riferimento anche al dibattito presente in letteratura sull'argomento (McMillan \& Schumacher, 2013). Di interesse indubbio è anche l'approfondimento del ruolo dell'esperienza nell'ambito dell'educazione comparata, che include anche i dibattiti sull'oggettività e sulla soggettività dei risultati rilevati rispettivamente con i metodi quantitativi o qualitativi.

$\grave{E}$ altresì noto che la realizzazione di ricerche comparative risente negativamente anche della logica della globalizzazione con cui vengono analizzate le differenti politiche educative nazionali, notoriamente variabili nei vari Paesi, regioni, comunità e istituzioni, che riducono fortemente la capacità delle ricerche attivate di dar conto degli intenti perseguiti. Ciò evidenzia l'esigenza diffusa di giungere alla conoscenza qualitativa e quantitativa dei fenomeni educativi tale da far emergere gli effetti della differenziazione e della interazione delle politiche e delle pratiche poste in atto, in modo tale da 
rendere i sistemi nazionali più equi in termini di accessibilità, di efficienza $\mathrm{e}$ di efficacia.

Come è noto, tale problematica è stata affrontata da tempo da autorevoli organizzazioni internazionali. Già nel 1990, infatti, i delegati di 155 Stati, nell'ambito della Conferenza promossa congiuntamente dalla Banca Mondiale, dall'UNESCO (United Nations Educational, Scientific and Cultural Organization), dall'UNICEF (United Nations Children's Fund) e dall'UNDP (United Nations Development Programme) hanno delineato lo stato dell'istruzione nel mondo evidenziando le condizioni spesso disastrose dei sistemi scolastici in molti Paesi, dove più di 100 milioni di giovani (il 60\% dei quali bambine) non avevano avuto, fino a quel momento, accesso all'istruzione primaria e circa 960 milioni di adulti erano analfabeti (Colbati, 2012).

Uno degli esiti della conferenza fu la sottoscrizione da parte dei delegati della World Declaration on Education for All (EFA) con il contestuale avvio della produzione di rapporti periodici sullo stato dell'istruzione dei Paesi interessati quali l'EFA Global Monitoring Report (EFA-GMR) ed il Global Education Digest (GED), entrambi dell'UNESCO, e anche di rapporti più sintetici quali il Millennium development goals Report, pubblicato periodicamente dalle Organizzazione delle Nazioni Unite (ONU).

Interessante sottolineare che, in tale contesto, in particolare dall'ultimo EFA-GMR dell'UNESCO (2012), è emerso che l'obiettivo di una istruzione primaria universale non sarà raggiunto nel 2015, come stabilito dalla conferenza di Dakar ${ }^{1}$ svoltasi nel 2000, in quanto nel 2010 risultavano iscritti a scuola circa 61 milioni di bambini, ovvero poco meno di 85 su 100. Questo dato, peraltro, non è completamente esaustivo, in quanto è stato elaborato senza aver potuto rilevare le informazioni di alcuni Paesi che, colpiti da gravi conflitti militari, non hanno reso possibile la raccolta di dati.

Inoltre ai fini comparativi, è altresì rilevante il contributo che l'OECD (Organization for Economic Cooperation and Development) fornisce nell'ambito del Report annuale Education at a glance, il primo dei quali pubblicato nel $2001^{2}$, in cui presenta sistematicamente indagini quantitative re-

1 Nel 2000 a Dakar (Senegal) 160 Paesi hanno partecipato al World Education Forum ed hanno deciso di adottare un ambizioso programma finalizzato ad ampliare le opportunità di apprendimento per i bambini, giovani e adulti, entro il 2015. Sono stati individuati 6 obiettivi (EFA goals): cura ed istruzione per l'infanzia; istruzione primaria universale; parità di genere; diffusione di alfabetizzazione degli adulti; programmi di ampliamento delle competenze per i giovani ed adulti; miglioramento della qualità dell'istruzione.

2 Il primo Report Education at a glance è stato pubblicato nel 1995, ma a partire dalla versione del 2001 sono presenti indicatori e paesi assenti in precedenza, tali da costituire significativi passi in avanti per la realizzazione di report maggiormente esaustivi rispetto a quelli delle precedenti edizioni. 
lative al funzionamento e alle risorse impegnate nei sistemi di istruzione dei Paesi OECD, nonché le valutazioni sulle performance degli studenti (2012). Riguardo quest'ultimo tema, risulta fondamentale l'indagine internazionale PISA (Programme for International Student Assessment), finalizzata a valutare su base periodica le conoscenze e le abilità dei quindicenni scolarizzati ${ }^{3}$.

\section{IL DIBATTITO SUL PROBLEMA DELLA COMPARABILITÀ DEI DATI NEGLI STUDI SULLE DISABILITÀ E SUI DSA: LINEE INTERPRETATIVE E PROSPETTICHE DELL'OECD E DELLA WHO}

È noto che l'assenza di una chiara ed univoca definizione di disabilità e di altri standard classificatori e procedurali ad essa relativi consente l'assunzione di criteri diversificati per il rilascio delle certificazioni e significativi margini di discrezionalità in fase di accertamento, di assegnazione di risorse e di assicurazione di garanzie specifiche. Il problema, come è stato già sottolineato, è interno ai diversi Paesi e si amplifica quando il campo di studio e di ricerca si estende al contesto internazionale.

Accade così che dalla scelta del modello teorico assunto a riferimento deriva l'individuazione degli indicatori adeguati e delle variabili che consentono di rendere il concetto di «disabilità» misurabile e quindi analizzabile.

In assenza di un piano internazionale uniforme, standardizzato e condiviso, i diversi sistemi di classificazione individuano e categorizzano le disabilità in modo differente da Paese a Paese e anche con una numerosità variabile di categorie considerate.

Questo stato di cose rende gli strumenti utilizzati molto diversi tra loro e, di conseguenza, i dati rilevati risultano scarsamente confrontabili in sede storica, anche all'interno dello stesso Paese, poco omogenei e non comparabili comunque a livello internazionale.

3 Il progetto PISA (Programme for International Student Assessment) è un'indagine internazionale promossa dall'OECD per accertare con periodicità triennale i risultati dei sistemi scolastici in un quadro comparato. La popolazione di riferimento è costituita dai quindicenni scolarizzati. Ogni ciclo dell'indagine approfondisce in particolare uno dei tre ambiti di competenza. Nella prima edizione, PISA 2000, il principale ambito di accertamento è stata la lettura, nella seconda edizione, PISA 2003 è stata la matematica, nella terza edizione, PISA 2006 le scienze, nella quarta edizione PISA 2009 l'ambito è stato nuovamente sulla lettura e la quinta edizione, PISA 2012, i cui risultati sono stati presentati nel dicembre 2013, ha riguardato le competenze in matematica e problem solving. La prossima rilevazione è prevista nel 2015 con le scienze come ambito principale.

ECPS Journal - 9/2014

http://www.ledonline.it/ECPS-Journal/ 
Dal momento che appare improbabile che le basi organiche della disabilità non siano riconosciute in modo condiviso e significativo tra i Paesi, è ipotizzabile, nell'ambito degli studi in corso per comprendere la complessità del fenomeno, che i divari siano connessi a criteri di classificazione collegati soprattutto alla concettualizzazione della disabilità, alle procedure di identificazione, alle pratiche educative, alla globalità dei provvedimenti messi in atto e alla priorità degli interventi previsti.

Per quanto riguarda i DSA, la problematicità risiede sia nelle differenze tra i sistemi linguistici nei vari Paesi, sia in alcune questioni cruciali tutt'ora aperte che riguardano, oltre la carenza di indagini epidemiologiche ampie (l'ultima risale al 1997) e la disponibilità di dati tratti quasi esclusivamente da riviste scientifiche (Tomblin et al., 1997), la collocazione dei quattro disturbi, dislessia, discalculia, disgrafia e disortografia, in categorie più generali come Learning disabilities ${ }^{4}$, Learning difficulties ${ }^{5}$ o Specific language impair-

4 Nel Giornale Italiano di Ricerca Clinica e Applicativa (Erickson, 2004, p. 100) sono riportate le seguenti definizioni: Per Kirk (1962), una learning disabilities si riferisce a «un ritardo, disordine o ritardato sviluppo in uno o più dei processi della parola, linguaggio, lettura, scrittura, aritmetica o altre aree scolastiche che risultano da un handicap psicologico causato da una possibile disfunzione cerebrale e/o disturbi emotivi o comportamentali. Non è il risultato di ritardo mentale, deprivazione sensoriale o fattori culturali e di istruzione». Per l'U.S. Office of Education (1977) «Il termine specific learning disabilities si riferisce a un disordine in uno o più processi psicologici di base implicati nella comprensione o nell'uso del linguaggio, parlato o scritto, che si può manifestare in una insufficiente capacità di ascoltare, parlare, leggere, esprimersi correttamente e adeguatamente per iscritto o fare calcoli matematici. Il termine include condizioni quali gli handicap percettivi, il danno cerebrale, la disfunzione cerebrale minima, la dislessia e l'afasia evolutiva. Il termine non include bambini che hanno disabilità di apprendimento che sono principalmente il risultato di handicap visivo, uditivo, motorio o mentale o di disturbo emotivo o di svantaggio ambientale, culturale, economico». Per Hammill (1990) «[...] learning disabilities è un termine generale che si riferisce ad un gruppo eterogeneo di disordini, manifestati da significative difficoltà nell'acquisizione e nell' uso di abilità di ascolto, espressione orale, lettura, scrittura, ragionamento e matematica. Questi disordini sono intrinseci all'individuo, presumibilmente dovuti a una disfunzione del sistema nervoso centrale, e possono verificarsi lungo l'arco della vita. Problemi nei comportamenti di autoregolazione, nella percezione sociale e nella interazione sociale possono coesistere con le learning disabilities. Anche se le disabilità di apprendimento possono verificarsi in concomitanza con altri fattori di handicap (per esempio, disturbo sensoriale, ritardo mentale, serio problema emotivo) o con influenze estrinseche (come differenze culturali, istruzione insufficiente o inappropriata) essi non sono il risultato di quelle condizioni o influenze».

5 Learning difficulties e learning disabilities sono termini che vengono comunemente utilizzati nel Regno Unito e spesso considerati intercambiabili. Nel campo dell'educazione il termine learning difficulties comprende bambini e giovani che hanno «specifiche difficoltà di apprendimento" come la dislessia, ma che non hanno una significativa compromissione dell'intelligenza. I codici che vengono utilizzati nell'ambito degli Special Educational Needs adottano termini che si riferiscono a situazioni dell'apprendimento con diversi livelli di gravità quali «difficoltà moderate di apprendimento»; «difficoltà di apprendimento grave»;

ECPS Journal - 9/2014

http://www.ledonline.it/ECPS-Journal/ 
$m e n t^{6}$. In relazione a tale orizzonte problematico che caratterizza tutt'ora i sistemi educativi europei, la prospettiva inclusiva significativamente promossa dalla WHO con l'ICF (International Classification of Functioning, Disability and Health) del 2001, incentrata sul concetto di human functioning in relazione al contesto di vita, offre uno sfondo integrato utile per interpretare e affrontare la complessità delle istituzioni scolastiche con allievi differenti tra loro e con tante singolari diversità, alcune delle quali sono oggetto di apposita certificazione, come nel caso delle disabilità e dei DSA.

Allo stesso modo, l'OECD, nel 2005, ha elaborato una classificazione che ha tenuto conto dei diversi approcci educativi nazionali sviluppando un sistema tripartito in cui la molteplicità e pluralità delle diversità/differenze sono ricondotte al concetto di Special Educational Needs (SEN), anche nell'intento di incidere sia a livello macro sull'impatto politico-culturale, sia su quello micro, sull'orientamento delle prassi educative e scolastiche nei vari Paesi.

Nonostante, dunque, il consenso internazionale sulla necessità di garantire il diritto all'istruzione a tutti i bambini e agli adolescenti (Convenzione ONU 2006; EFA 2009; HORIZON 2020) prescindendo dal sistema delle certificazioni, non è stato ancora raggiunto quel grado di univocità necessaria a definire e interpretare la disabilità e i DSA che possa permettere di rilevare dati affidabili e confrontabili, indispensabili per lo sviluppo reale dei processi di inclusione in ciascun Paese e l'esercizio attivo dei diritti fondamentali.

Di grande rilievo, in tale direzione, sono dunque le prospettive della WHO e dell'OECD rispetto ad approcci scientifici e unitari al problema, progressivamente sviluppate sulla base degli esiti delle ricerche di settore e, soprattutto, del dibattito nazionale e internazionale, sia per le suggestioni culturali sia per la visione a forte vocazione unitaria che possono contribuire a costruire rispetto alla diversità.

Se, infatti, i SEN sono analizzati, interpretati e valutati in relazione alla prospettiva inclusiva della WHO e, di conseguenza, considerati nella loro stretta interazione con i fattori ambientali, può delinearsi una matrice classificatoria di grande interesse scientifico, teorica e applicativa, in grado di rinnovare la cultura dell'inclusione favorendo nello stesso tempo l'impiego di un linguaggio universale per la produzione di dati utili sul piano della condivisione, diffusione e comparazione. Ciò sembrerebbe poter effettivamente rilanciare quella collaborazione di ampio respiro culturale, scientifico, sociale

«difficoltà di apprendimento multipla». Per ulteriori approfondimenti si rimanda ai seguenti siti: www.improvinghealthandlives.org.uk; www.bild.org.uk; www.bris.ac.uk/cipold.

6 Per approfondimenti cfr. il sito dell'U.S. Department of Health \& Human Services in http://www.hhs.gov. 
e istituzionale, tanto auspicata quanto necessaria, in grado di dare risposte al diritto all'accesso all'istruzione e alla formazione e al successo di ciascun alunno secondo le individuali potenzialità da sviluppare al massimo grado possibile verso competenze trasversali e/o specifiche nei vari contesti.

\section{INDAGINI QUANTITATIVE NEGLI STUDI DEI DSA E DELLE DISABILITÀ IN AMBITO INTERNAZIONALE: I SEN NEL SISTEMA TRIPARTITO DELL'OECD («Disability, Difficulty, Disadvantage» - DDD)}

Come è stato anticipato nel paragrafo precedente, l'OECD considera le disabilità e i DSA all'interno di un unico sistema codificatorio, articolato in tre parti, incentrato sulla concettualizzazione del significato di Special Educational Needs. Prima di darne conto, seppure sinteticamente in queste pagine, è opportuno richiamare due traiettorie di riflessione culturale e scientifica, storicamente rilevanti.

La prima è relativa al fatto che a tali «bisogni educativi speciali» (SEN/ BES) si fa riferimento fin dal 1978 nel Rapporto Warnock in cui si precisa che essi «riguardano allievi con qualsiasi difficoltà di tipo evolutivo nel funzionamento del soggetto dal punto di vista educativo e dell'apprendimento e che presentano uno stato di difficoltà in cui può venirsi a trovare un bambino, un preadolescente o un adolescente a causa di differenti problematiche personali e sociali, che non sono causate esclusivamente da una disabilità» (HMSO-Warnock, 1978, p. 37).

La seconda riguarda invece l'elaborazione di Booth e Ainscow nel 2000, pubblicata dal Centre for Studies on Inclusive Education in Gran Bretagna (trad. it., 2008), che, con la lettura critica del modello biomedico della disabilità, ha rappresentato un punto di svolta fondamentale verso un approccio inclusivo di tutte le diversità, e non solo delle disabilità, basato sull'individuazione di obiettivi formativi comuni a tutti gli alunni, indipendentemente dal livello di abilità, e in grado di far fronte alla complessità delle richieste provenienti dagli alunni.

I due studiosi sostengono infatti che, nell'ottica dell'inclusione, appare riduttivo fare riferimento esclusivamente ad alunni che presentano problemi fisici o mentali, o più in generale bisogni educativi speciali. Sarebbe invece da ripensare l'idea di inclusione fondandola sulla promozione dell'apprendimento per tutti e sulla piena partecipazione alla vita scolastica di ciascuno, al fine di realizzare una comunità in grado di accogliere pienamente anche mediante una trasformazione del proprio curriculum e delle strategie organiz- 
zative e didattiche per renderle rispondenti all'intera gradazione delle diversità in essa presenti.

Ma a fronte di tali pur significative cornici interpretative, non facilmente coniugabili tra loro, le definizioni proprie dei bisogni educativi speciali variano da un Paese all'altro in relazione alle specifiche normative vigenti in materia e delle peculiari valenze nazionali attribuite ad essi (OECD, 2012a) ${ }^{7}$.

Al fine di poter effettuare studi comparativi riguardanti i SEN/BES, l'OECD già nel 2005 aveva infatti individuato gli allievi con SEN sulla base della classificazione ISCED 1997, rivisitata nel 2011 (International Standard Classification of Education) ${ }^{8}$ cui fa riferimento '’UNESCO $^{9}$, come «coloro i

7 OECD - Social Policy Division - Directorate of Employment, Labour and Social Affairs, in Child well-being Module http://www.oecd.org/els/social/family/database/CWBM, May 2012. CX3.1 Special Educational Needs (SEN).

8 ISCED 2011 utilizzato a partire dal 2014 è una revisione dello schema ISCED 1997. La revisione è stata approvata dalla Conferenza generale dell'UNESCO ed è reperibile all'indirizzo: http://www.uis.unesco.org/Education/Documents/UNESCO_GC_36C-19_ ISCED_EN.pdf. I criteri dello schema ISCED 1997, usato fino al 2011, sono descritti nè sito dell'UIS all'indirizzo: http://www.uis.unesco.org/Library/Documents/isced97-en.pdf. ISCED 1997 è articolato in sette livelli: pre-primario, livello 0 (da 3 anni di età in poi); primario, livello 1 (primo stadio dell'istruzione di base, 6 anni al massimo); a livello secondario si distingue tra un livello inferiore 2 , detto anche di secondo stadio di istruzione di base, e uno superiore, livello 3; al di sopra del secondario, tra un post-secondario non terziario, livello 4 e due livelli di formazione terziaria: inferiore (5) e superiore (6). ISCED 2011 ha introdotto delle novità: a livello 0 (pre-scuola) si distingue tra 01 (nei primi due anni di vita) e 02 (dal terzo anno di vita in poi); i livelli 1, 2, 3 e 4 corrispondono sostanzialmente ai livelli corrispondenti di ISCED 1997; il livello 4 è per l'istruzione post-secondaria non terziaria, cioè avente carattere maggiormente professionalizzante. La novità maggiore riguarda l'istruzione terziaria, divisa in quattro livelli. Il primo, 5, porta con un ciclo breve di almeno 2 anni direttamente al mercato del lavoro; il livello «intermedio" (6) è quello del bachelor, con 4 o più anni: qui il contenuto teorico è più elevato e i docenti devono essere di livello 7 od 8 ; il livello 7 vede aumentare la durata (livello di master) e contenuto teorico; qui sono compresi i programmi che danno accesso al livello 8 , che riguarda i «dottori di ricerca». La classificazione dei 7 campi dell'istruzione è rimasta invariata: Basic programmes; Literacy and numeracy; Personal development; Teacher training and education science; Education science; Humanities and arts; Journalism and information. Per la prima volta accanto a questa classificazione figura una che tiene conto dell'educational attainment, cioè dell'ottenimento o meno (per frequenza incompleta) di un titolo.

9 UNESCO (2011), Revision of the International Standard Classification of Education (ISCED). Gli Special Education Needs riguardano «l'educazione che viene progettata per facilitare la possibilità di conseguire gli obiettivi di apprendimento di un programma educativo a persone che, per diversi motivi, necessitano di un sostegno aggiuntivo e di metodi pedagogici adattivi. I motivi riguardano principalmente, ma non solo, svantaggi fisici, intellettivi, emozionali e capacità sociali. I programmi di formazione nell'educazione speciale possono seguire un curriculum simile a quello offerto nel sistema di istruzione tradizionale, ma possono tener conto delle particolari esigenze delle persone prevedendo specifiche risorse (ad esempio personale qualificato, attrezzature o spazi) e, se necessario, la modifica dei contenuti educativi o 
quali non possono beneficiare dell'istruzione scolastica prevista per alunni della stessa età senza che vengano attivate risorse aggiuntive (pubbliche o private) tali da aiutare gli alunni in difficoltà ad accedere più efficacemente al programma educativo" (OECD, 2005, p. 3). Naturalmente le risorse indicate dall'OECD, non riguardano esclusivamente quelle finanziarie, ma possono riferirsi a risorse professionali specializzate, all'organizzazione didattica dei tempi e degli spazi di apprendimento, a materiali specifici, a supporti ed ausilii tecnologici.

Sulla base di tale definizione di «risorse», al fine di rilevare dati comparativi tra i diversi Paesi, l'OECD ha ricondotto l'ampia varietà delle categorizzazioni impiegate in ciascuno di essi alle seguenti tre macro categorie sovranazionali (Disability, Difficulty, Disadvantage - DDD) ${ }^{10}$.

a. Alunni con disabilità (Categoria $\mathrm{A}-$ Disability): disabilità attribuibili a patologie organiche (in relazione a problemi sensoriali, motori o neurologici). Le specifiche esigenze formative dipendono principalmente da problemi attribuibili a queste disabilità.

b. Alunni con difficoltà (Categoria B - Difficulty): difficoltà determinate da problemi emotivi e comportamentali o disturbi specifici dell'apprendimento (DSA). I problemi principali nascono dall'interazione fra l'alunno e il contesto educativo.

c. Alunni svantaggiati (Categoria C - Disadvantage): svantaggi causati da un background socio-economico problematico o semplicemente differente sotto l'aspetto linguistico e/o culturale da quello delle classi di accoglienza (ad esempio i bambini stranieri). Il bisogno educativo è quello di compensare gli svantaggi attribuibili a tali fattori.

Un'importante applicazione di tali categorie in ottica comparativa ha riguardato la possibilità di valutare, in particolare, la percentuale di alunni della Categoria A, B e C cui sono state destinate risorse aggiuntive nei Paesi OECD considerati, come riportato nelle seguenti figure (Figure 1-3).

In sintesi, rispetto al quadro rappresentato, le maggiori problematiche emergono nel tentativo di disporre di studi comparativi a livello internazionale per l'individuazione di alunni con DSA, a causa della mancanza di un linguaggio standard in grado di rilevarli univocamente.

degli obiettivi di apprendimento. I programmi possono essere previsti o per i singoli studenti nell'ambito di programmi educativi già esistenti, o possono essere organizzati come una classe distinta nella stessa scuola o possono essere previste in specifiche istituzioni scolastiche» (p. 83).

${ }_{10}$ I dati presentati per ciascuna categoria (disabilità, difficoltà, svantaggio), variamente utilizzate nei vari contesti nazionali, sono ripartiti in base al paese in cui gli alunni ricevono risorse aggiuntive e si concentrano sugli anni della scuola dell'obbligo, che in genere è da circa 6-16 anni in molti paesi. I dati relativi alla scuola secondaria riguardanti gli studenti con disabilità, difficoltà o svantaggi (DDD), risultano in generale meno disponibili. 


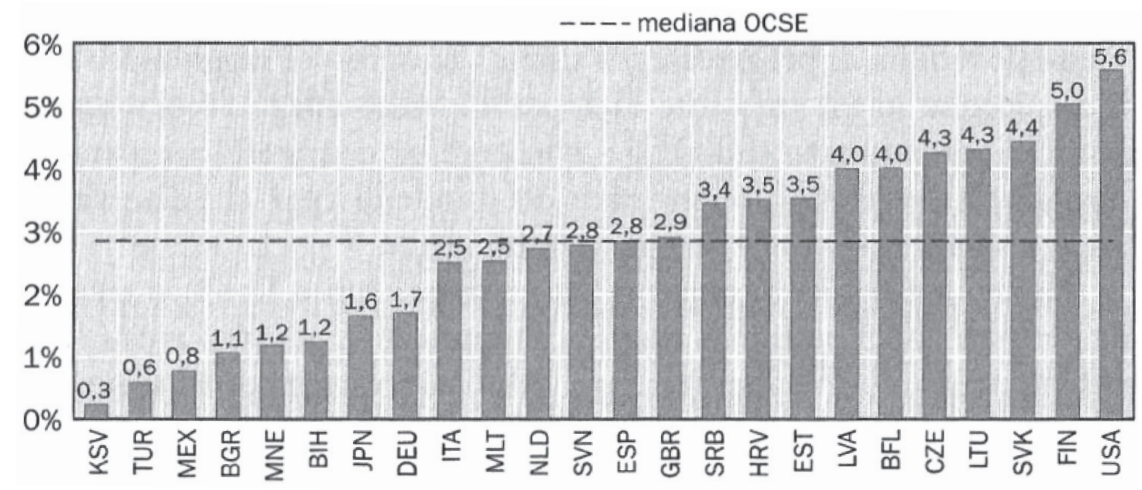

Figura 1. - Alunni con disabilità (Categoria A) nella scuola dell'obbligo a cui vengono destinate risorse aggiuntive (2005): percentuale sul totale degli alunni della scuola dell'obbligo.

Fonte: OECD SENDDD Database.

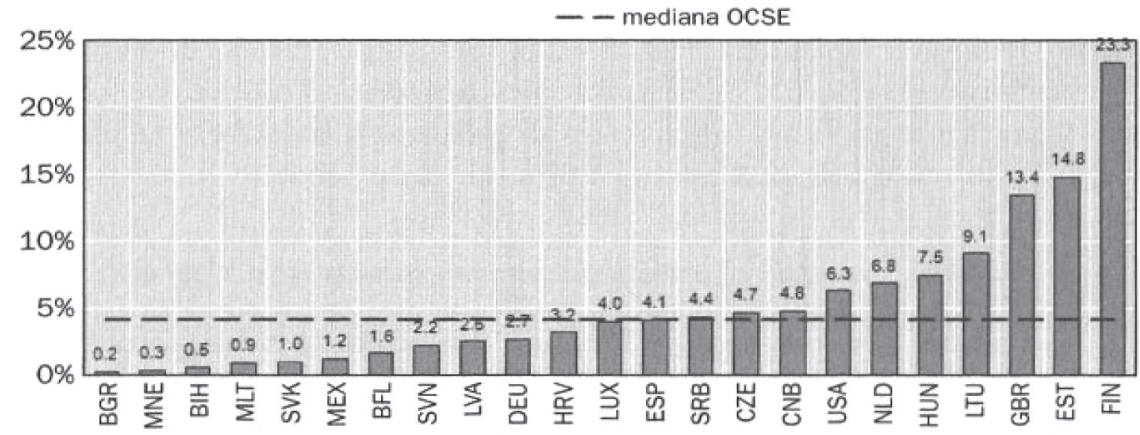

Figura 2. - Alunni con difficoltà (Categoria B) nella scuola dell'obbligo a cui vengono destinate risorse aggiuntive (2005): percentuale sul totale degli alunni della scuola dell'obbligo.

Fonte: OECD SENDDD Database.

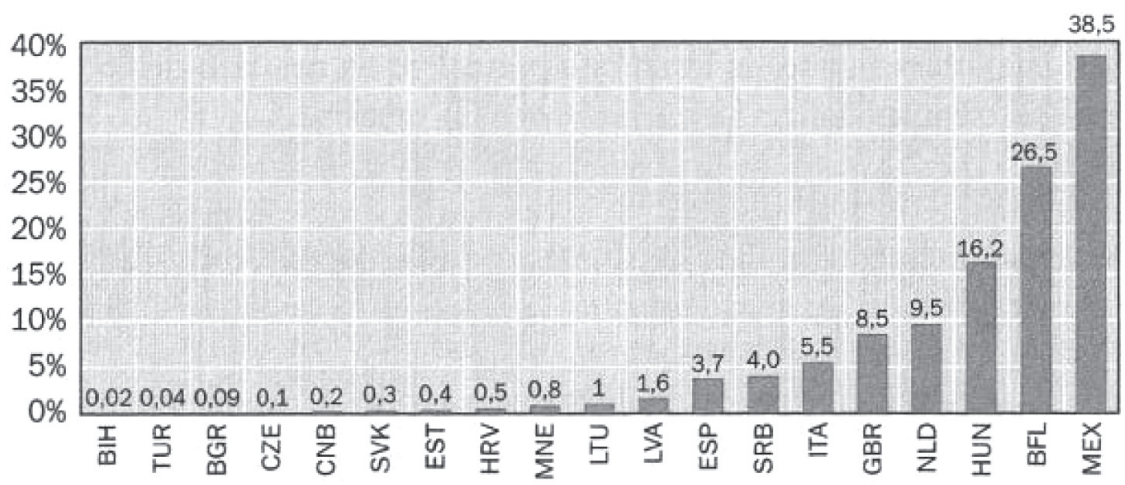

Figura 3. - Alunni con svantaggi (Categoria C) nella scuola dell'obbligo a cui vengono destinate risorse aggiuntive (2005): percentuale sul totale degli alunni della scuola dell'obbligo. 
Ad esempio, mentre in Italia, con la Legge n. 170 del 2010, i DSA sono stati riconosciuti e definiti nella dislessia, disgrafia, disortografia e discalculia e «si manifestano in presenza di capacità cognitive adeguate, in assenza di patologie neurologiche e di deficit sensoriali, ma possono costituire una limitazione importante per alcune attività della vita quotidiana» (art. 1, p. 1), in ambito internazionale i DSA rientrano in categorie più ampie e generali facenti capo alle già citate aree di ricerca delle Learning disabilities, delle Learning difficulties o di quella relativa agli Specific language impairment, riferiti in particolare ai disturbi del linguaggio.

La difficoltà di individuare categorie specifiche dipende infatti da molteplici fattori, citati in precedenza, tra i quali spiccano la prevalenza di studi internazionali sulla dislessia e la carenza di quelli sulla disgrafia, disortografia e discalculia e la modesta attività di ricerca, rinvenibile pressoché esclusivamente su riviste scientifiche. In riferimento a quest'ultimo aspetto, Tomblin (1997) riporta i risultati di uno studio epidemiologico relativo all'individuazione dello Specific language impairment che ha riguardato un gruppo di bambini monolingue di una scuola dell'infanzia inglese. Su 7218 bambini sottoposti allo screening, il $17,4 \%$, di cui il $10 \%$ maschi e il $7,4 \%$ femmine, risultava avere un disturbo specifico del linguaggio.

\section{INDAGINI QUANTITATIVE NEGLI STUDI SULLE DISABILITÀ IN AMBITO INTERNAZIONALE: IL CONTRIBUTO DI UNICEF, UNESCO E WHO}

Secondo una stima molto diffusa, circa 93 milioni di bambini e di adolescenti, di cui il 5\% con meno di14 anni, convivono con una disabilità di grado medio o grave. Si tratta di «stime internazionali che derivano da dati di qualità abbastanza variabile e da metodi troppo discontinui per essere affidabili» (UNICEF, 2013, p. 3) che, in riferimento al nostro Paese e non solo, hanno un impatto rilevante sulla possibilità di realizzare processi scolastici e sociali inclusivi. Tale fragilità di stime va a sommarsi ad altre presenti nei sistemi educativi, quali la carenza di formazione di tutti gli insegnanti, l'impegno collettivo nella costruzione di risposte condivise ed adeguate alle esigenze individuali degli allievi, la qualità dei programmi, la rigidità dei curricoli e delle procedure valutative, la perdurante predominanza dell'approccio medico alla disabilità, la frequenza a volte molto frammentaria e discontinua degli allievi disabili nelle classi di appartenenza, nonché la carenza di collaborazione tra l'insegnante di sostegno e gli insegnanti su posto comune (Chiappetta Cajola, 2004 e 2007). 
La possibilità, invece, di disporre di informazioni e dati quantitativi di ricerca sulla disabilità ${ }^{11}$ che siano affidabili e comparabili e di poter utilizzare un linguaggio per codificarli e interpretarli in modo univoco, rappresenta un presupposto fondamentale anche per la corretta attuazione del dettato legislativo in materia di integrazione, nonché per l'assegnazione di risorse, umane e materiali, adeguate alla prospettiva inclusiva e alla complessità del contesto sociale in cui la scuola si trova ad operare.

Se, dunque, si intende comparare tra loro i sistemi scolastici a livello internazionale rispetto all'integrazione degli allievi con disabilità, è indispensabile disporre di una definizione univoca del concetto di disabilità nonché di uno strumento che permetta di rilevare e analizzare con modalità altrettanto univoche le caratteristiche inclusive della scuola: i livelli di istruzione, le figure professionali, quali il dirigente scolastico, l'insegnante su posto comune o per le attività di sostegno, i programmi dell'istruzione.

A tali nodi cruciali ben corrispondono gli strumenti forniti dall'Organizzazione Mondiale della Sanità (WHO) con la versione generale dell'International Classification of Functioning, Disability and Health (2001), successivamente integrata con la versione per bambini ed adolescenti, ICF-CY (International Classification of Functioning, Disability and Health for Children and Youth, 2007), nonché dai criteri di classificazione dell'istruzione ISCED 2011 (International Standard Classification of Education) ${ }^{12}$ progettata dall'UNESCO.

11 La domanda di statistiche sul funzionamento umano e la disabilità è notevolmente aumentata dopo l'Anno internazionale delle persone disabili (1981), l'adozione del Programma Mondiale delle Nazioni Unite di azione per le persone disabili (1982), e il rilascio delle Regole Standard per le pari opportunità delle persone con disabilità (1993). In particolare, il Programma Mondiale di Azione espressamente richiedeva alle Nazioni Unite di sviluppare sistemi per la regolare raccolta e diffusione di informazioni sulla disabilità. Il questionario sul funzionamento umano e le statistiche relative alla disabilità (parte 1 e parte 2 del documento) sono stati successivamente sviluppati in base alle raccomandazioni e alle decisioni messe a punto da gruppi di esperti e dalla Commissione Statistica delle Nazioni Unite. Nel marzo 2006 la Divisione Statistica delle Nazioni Unite ha avviato una raccolta di statistiche di base sulla disabilità attraverso il sistema di raccolta Demographic Yearbook, che rappresenta una guida per le norme e i metodi dei dati disponibili sul funzionamento umano e disabilità. Importante è l'azione del DISTAT (The United Nations Disability Statistics Database). Ha sottolineato che, a causa delle differenze nei concetti e nei metodi usati per identificare le persone con disabilità, le percentuali non devono essere confrontate tra i vari Paesi. Altresì importante è il Disability Statistics Compendium, pubblicato per la prima volta nel 1990, sulla base delle statistiche nazionali disponibili in DISTAT.

12 ISCED 2011, come descritto nella nota 9, utilizzato a partire dal 2014 è una revisione dello schema ISCED 1997. La revisione è stata approvata dalla Conferenza generale dell'UNESCO ed è reperibile all'indirizzo: http:// www.uis.unesco.org/Education/ Documents/UNESCO_GC_36C-19_ISCED_EN.pdf. I criteri dello schema ISCED 1997, usato fino al 2011, sono descritti nel sito dell'UIS all'indirizzo: http://www.uis.unesco.org/ Library/Documents/isced97-en.pdf. 
Attualmente l'ICF, il cui scopo generale è proprio quello di «fornire un linguaggio standard e unificato che serva da modello di riferimento per la descrizione della salute e degli stati ad essa correlati» (WHO, 2001, p. 11), rappresenta «l'unico strumento del genere ad essere accettato internazionalmente. Esso mira ad ottenere migliori informazioni sui fenomeni della disabilità $\mathrm{e}$ del funzionamento, nonché a raggiungere un vasto consenso internazionale. Per fare in modo che l'ICF fosse riconosciuto da varie comunità nazionali ed internazionali, la WHO ha fatto tutto il possibile per renderlo di facile utilizzo e compatibile con processi di standardizzazione come quelli indicati dalla International for Standardization (ISO)» (ivi, p. 198).

Con l'ICF, l'impegno della WHO è quello di riuscire a evidenziare, superando l'impostazione del modello medico, la multifattorialità di cui la disabilità si compone, attraverso la descrizione e la misurazione dell'influenza ambientale sulla persona e sul suo "funzionamento", facendo così emergere la centralità del contesto nella definizione stessa di disabilità ${ }^{13}$.

Ciò significa che ad un individuo con disabilità, nel momento della certificazione, viene conclamata oggettivamente una discrepanza di funzionamento rispetto ad una norma; soggettivamente, però, il percorso di vita successivo è fondamentalmente condizionato dal contesto che incontra vivendo.

In questa ottica, la WHO sostiene che la salute non può essere concepita come semplice assenza di malattia, ma va pensata e soprattutto perseguita come globale benessere bio-psico-sociale dell'individuo posto nelle condizioni di poter sviluppare compiutamente i propri potenziali in interazione con $\mathrm{i}$ fattori ambientali ${ }^{14}$ presenti nei vari contesti di vita, che possono agire nega-

13 Già nel 1980 la WHO, nel tentativo di ricercare concetti, definizioni e terminologie quanto più omogenei e condivisibili, pubblicò l'International Classification of Impairments, Disabilities and Handicaps (ICIDH) dove veniva introdotta l'importante distinzione fra «menomazione» (impairment), "disabilità» (disability) e "handicap", anche se con un'evidente limitazione dovuta alla interpretazione sequenziale dei tre eventi: chi ha una malattia o un disturbo, ha un danno organico (menomazione), di conseguenza ha una perdita di capacità operative (disabilità) e quindi uno svantaggio sociale (handicap). Inoltre i termini menomazione, disabilità e handicap erano usati in accezione negativa, con riferimento a situazioni di deficit. La decima revisione dell'ICIDH, avviata dalla WHO nel 1993, ha condotto al superamento di questo modello con la stesura dell'ICF che permette di passare da un modello sequenziale ad uno reticolare e sistemico, nel quale per la prima volta viene effettuata una classificazione sistematica dei fattori ambientali e dove la disabilità assume il valore di una condizione di salute in un ambiente sfavorevole. Anche dal punto di vista linguistico si assiste ad un cambiamento notevole: non vengono più utilizzati termini quali «menomazione» $\mathrm{o}$ "handicap», ritenuti lesivi per la dignità della persona a causa di una loro connotazione negativa, oltre che riduttivi della persona, ma si adopera invece un linguaggio più corretto e condivisibile.

14 I fattori ambientali vengono definiti nell'ICF come «gli atteggiamenti, l'ambiente fisico e sociale in cui le persone vivono e conducono la loro esistenza» (WHO, 2007, p. 20). L'interazione persona-ambiente richiede di prestare una particolare attenzione ai fattori ambientali 
tivamente come «barriere» determinando la disabilità o positivamente come «facilitatori» determinando uno stato di salute.

Da questo punto di vista, la dimensione universale dell'ICF emerge nella misura in cui la disabilità, determinata da una condizione ambientale sfavorevole, non viene considerata un problema di un gruppo minoritario all'interno di una comunità, ma un'esperienza che tutti, nell'arco della vita, possono sperimentare.

Tale concezione dinamica e interattiva della disabilità, recepita pienamente dalla Convenzione ONU del 2006 riguardante i diritti delle persone con disabilità (Convention on the Rights of Persons with Disabilities - CRPD), palesa anche la necessità di introdurre modelli di valutazione e intervento sulla disabilità ispirati proprio ai contenuti della Convenzione stessa, attraverso in particolare la promozione dei diritti umani, l'inclusione sociale, l'eliminazione delle barriere presenti nell'ambiente e promuovendo massimamente i facilitatori in grado di contrastare anche la discriminazione e l'impoverimento.

Si tratta sostanzialmente di valorizzare gli aspetti positivi del sistema attuale di welfare, innovandoli, e di ricondurre ad unità la frammentazione normativa e delle prassi esistente, attraverso il raggiungimento dei principali obiettivi della WHO che sono diretti essenzialmente a:

- sviluppare e diffondere la cultura dell'inclusione attraverso la visione del "funzionamento umano» che è a fondamento dell'ICF, promuovendo cambiamenti significativi dei vari contesti;

- utilizzare le componenti dell'ICF (funzioni e strutture corporee; attività e partecipazione; fattori contestuali) come base per la costruzione di strumenti che, oltre all'ambito clinico, possano essere efficaci in ambito statistico per indagini quantitative, di ricerca e di formazione (cfr. WHO, 2002, p. 13), ma anche come strumento di politica sociale per orientare l'elaborazione di programmi specifici, quali ad esempio quelli di previdenza sociale, nonché per le ricadute su ampi settori quali l'istruzione, il lavoro, la legislazione, l'economia, in modo tale da contribuire a definire gli interventi che possano garantire uguaglianza di accesso ai funzionamenti più attinenti

nel caso dei bambini e degli adolescenti. Infatti gli ambienti dei bambini e degli adolescenti possono essere considerati come una serie di sistemi successivi che li circondano, dal più immediato al più distante, aventi ognuno un'influenza diversa, che varia in funzione dell'età o dello stadio evolutivo raggiunto. In considerazione della posizione di dipendenza in cui si trovano i bambini durante lo sviluppo, gli elementi fisici e sociali dell'ambiente hanno un impatto significativo sul loro funzionamento pertanto i fattori ambientali negativi hanno spesso un impatto più forte sui bambini che sugli adulti; per gli adolescenti, si diversificano gradualmente nel contesto più ampio della comunità e della società. I fattori personali non sono codificati perché propri di ciascuna persona. 
rispettivamente all'attività (prospettiva individuale del funzionamento) e alla partecipazione ai contesti di vita (prospettiva sociale del funzionamento), quali i piani assistenziali, i progetti individualizzati/personalizzati.

\subsection{WHO e il primo "World Report on Disability» in linguaggio ICF}

In relazione agli obiettivi appena elencati e sulla base del quadro concettuale dell'ICF, l'Organizzazione Mondiale della Sanità ha realizzato nel 2011 il primo Rapporto Mondiale della Disabilità (World Report on Disability) nell'intento esplicito, tra gli altri, di ottenere informazioni in merito allo stato di avanzamento dell'implementazione, da parte degli Stati, di politiche e programmi finalizzati a migliorare le condizioni di vita delle persone con disabilità in linea con quanto stabilito dalla CRPD del 2006. L'indagine ha coinvolto 70 Paesi a livello mondiale, ed il confronto tra i dati rilevati è stato reso possibile proprio grazie all'utilizzo della Classificazione ICF che, con le sue definizioni standard ed universali, ha consentito di rilevare gli aspetti delle menomazioni relative alle funzioni e strutture corporee, le eventuali limitazioni nello svolgimento delle attività e le restrizioni alla partecipazione sociale e gli impatti, facilitanti o ostacolanti, dei fattori ambientali.

Lo strumento di raccolta dei dati era strettamente sincronizzato con la progressiva revisione della Classificazione Internazionale del Funzionamento, della Disabilità e della Salute (ICF) così come indicato nelle Guidelines and principles for the development of disability statistics (WHO, 2001) ${ }^{15}$. Il lavoro condotto dalla WHO rappresenta il primo contributo nel suo genere, in quanto fornisce una guida globale per l'attuazione della CRPD e rappresenta un ampio quadro delle esigenze delle persone con disabilità, dei bisogni insoddisfatti e degli ostacoli che devono affrontare per poter partecipare attivamente a tutto ciò che attiene la vita quotidiana.

L'utilizzo dell'ICF (e dell'ICF-CY), anche se non completamente generalizzato nelle rilevazioni di tutti i Paesi e nello stesso periodo temporale ${ }^{16}$, ha

15 La pubblicazione ha lo scopo di assistere gli uffici nazionali di statistica e gli altri produttori di statistiche relative alla disabilità per migliorare la raccolta, la compilazione e la diffusione di dati. Il documento affronta questioni metodologiche nel campo della disabilità, fornendo linee guida e principi relativi alla raccolta dei dati, attraverso indagini e censimenti e anche nella compilation, la diffusione e l'utilizzo di dati sulla disabilità. Interviene su questioni legate all'elaborazione di statistiche e di indicatori sulla situazione delle persone con disabilità e propone l'uso dell'ICF in quanto in grado di supportare processi statistici confrontabili, stante la varietà di modelli concettuali e metodi utilizzati dai vari paesi.

16 Solo in alcuni paesi sono stati rilevati gli aspetti delle attività, delle restrizioni alla partecipazione e gli impatti dei fattori ambientali; mentre in altri paesi solo una parte di questi 
senz’altro contribuito ad ottenere informazioni sulla presenza delle persone con disabilità e sulle loro esigenze e necessità con maggior precisione rispetto alle stime prodotte sempre dalla WHO con il metodo del Global Burden of Disease (GBD) ${ }^{17}$. Anche se con le limitazioni riscontrate nelle metodologie dei singoli Paesi coinvolti (World Report on Disability, 2011), è emerso che circa 785 milioni $(15,6 \%)$ di persone con almeno 15 anni di età presentano una disabilità (il Global Burden of Disease stima la cifra di circa 975 milioni pari al 19,4\% di persone); di queste 110 milioni delle disabilità di persone $(2,2 \%)$ sono molto significative (il Global Burden of Disease ne stima circa 190 milioni ovvero il 3,8\%). Solo il Global Burden of Disease misura disabilità infantili (0-14 anni): i bambini sono 95 milioni $(5,1 \%)$ di cui 13 milioni $(0,7 \%)$ hanno una "disabilità grave», considerata tale per determinate condizioni come ad esempio la tetraplegia, depressione grave, o cecità.

\subsection{Il concetto di «funzionamento umano» e di «capacità di funzionare» nell'ICF}

Per la rivisitazione innovativa dei concetti di «funzionamento umano / salute / disabilità" che propone, l'ICF rappresenta attualmente il linguaggio descrittivo standard più evoluto e condiviso, e permette di conseguenza ad operatori appartenenti ad ambiti differenti, ma ugualmente coinvolti nel processo di integrazione, di comunicare con immediatezza e precisione.

Tale linguaggio rappresenta in modo adeguato il modello antropologico, biopsicosociale, proprio dell'ICF, caratterizzato dall'approccio alla persona coerente con il principio della dignità umana, con il diritto allo sviluppo delle proprie potenzialità e all'esercizio di tutti i diritti fondamentali in un contesto di vita intenzionalmente adattato, e reso adatto a tali fini (Chiappetta Cajola, 2012 e 2013).

A ben vedere, si potrebbe parlare più opportunamente dell'esercizio di un meta-diritto inviolabile quale è quello di «poter usufruire dei diritti» e di poterli esercitare, che è allo stesso tempo anche un diritto a condizioni di-

fattori. I periodi di rilevazione variano dal 2001 al 2008, a seconda del paese analizzato. Per visualizzare il dettaglio per paese partecipante all'indagine cfr. "Technical Appendix A», nel World Report on disability (2011).

17 Il Global Burden of Disease del WHO fornisce stime sulla disabilità mediante una valutazione che misura "il peso» o "l'impatto» della malattia utilizzando il Disability-Adjusted Life Year (DALY). Questa misura valuta gli anni di vita persi a causa di mortalità prematura e gli anni di vita persi a causa delle condizioni vissute non in piena salute. La metrica DALY è stata sviluppata dal GBD nel 1990 per valutare uniformemente l'impatto delle malattie ed i fattori di rischio nei paesi a livello internazionale. Per approfondimenti cfr. http://www.who. int/topics/global_burden_of_disease/en. 
gnitose di esistenza e alla riduzione delle disuguaglianze, stati umani imprescindibili per realizzare pari opportunità di vivere in modo complessivamente corrispondente all'obiettivo della dignità umana.

Quest'ultima rappresenta infatti il presupposto della relazione con l'altro e del riconoscimento reciproco ed è garanzia, nell'eguaglianza che nasce dalla comune dignità, del rispetto delle diversità e nel contempo dell'impegno alla eliminazione degli ostacoli che, nell'ambiente di vita, trasformano le differenze in condizioni di inferiorità.

$\grave{E}$ in questa prospettiva che si può comprendere appieno quanto la dignità sia impossibile senza solidarietà che diviene quindi un valore unificante: non può esservi uguaglianza delle opportunità senza solidarietà, poiché solo la solidarietà è in grado di superare la diversità nella prospettiva dell'eguaglianza.

Da qui l'importanza delle sinergie solidali che diano spazio a un continuum di confronto e collaborazione favorito dalla condivisione linguistica e scientifica e dalla medesima base conoscitiva dei fenomeni che permettono sia un dialogo significativo sia l'uso oggettivo di strumenti finalizzati ad identificare quel «funzionamento" di una persona, interpretandone i diversi gradi di definizione e la misurazione in riferimento a una norma o a soglie stabilite su base statistica.

A. Sen definisce i functioning come "le opportunità di vita» (Sen, 1992, p. 62), influenzate dalle capabilities individuali di attivare determinati functioning. Con il termine capabilities Sen intende la "capacitazione» dell'individuo e non la capacità comunemente intesa. Si tratta di una distinzione fondamentale perché con capabilities vengono indicati non solo gli stati di essere propri della persona, le sue caratteristiche, ma anche le capacità che una società dà o nega all'individuo.

È questa dimensione complessa del capability approach che rende evidente la consonanza dei principi seniani con l'ICF nel momento in cui il funzionamento umano viene identificato con il risultato dell'interazione tra le caratteristiche dell'individuo a livello delle proprie strutture e funzioni corporee, e i fattori personali e ambientali, e con la conseguente «capacità di funzionare».

Risultato che non è definitivamente raggiunto, in quanto la maggior parte delle capacità atte a realizzare determinati functioning non sono date una volta per tutte in un individuo, ma sono piuttosto legate ad avvenimenti, eventi, situazioni critiche di vita, problemi di varia origine che possono farle perdere temporaneamente $\mathrm{o}$ in maniera permanente, riducendo «le opportunità di vita».

Sostiene Sen che «si può pensare che la vita consista in un insieme di funzionamenti composti di stati di essere e di fare. I funzionamenti rilevanti 
possono variare da cose elementari come essere adeguatamente nutriti, essere in buona salute, sfuggire alle morbilità prevenibili [...] fino ad acquisizioni più complesse come essere felice, avere rispetto di sé, prendere parte alla vita della comunità e così via». La tesi di fondo è che i funzionamenti siano costitutivi dell'essere di una persona, e che una valutazione dello star bene debba prendere la forma di un giudizio su tali elementi costitutivi. Strettamente legata alla definizione di funzionamento è quella di "capacità di funzionare». Essa rappresenta le varie combinazioni di funzionamenti (stati di essere e di fare) che la persona può acquisire. "La capacità è dunque un insieme di vettori di funzionamenti, e riflette la libertà dell'individuo di condurre un certo tipo di vita piuttosto che un altro» (Sen, 1992, pp. 63-64).

In tale quadro, e alla luce di quanto fin qui argomentato, l'ICF è rivolto a tutti, e sembra fornire anche una chiave di lettura dei «Bisogni Educativi Speciali» non disgiunti dagli «ostacoli all'apprendimento e alla partecipazione», nel tentativo implicito di superare in senso dialettico la contrapposizione tra le due aree concettuali.

La centralità del significato di «funzionamento umano» diviene cruciale anche per orientare verso l'uguaglianza di accesso ai funzionamenti rilevanti e più attinenti all'attività (prospettiva individuale del funzionamento) e alla partecipazione (prospettiva sociale del funzionamento).

Da questo punto di osservazione, il modello biopsicosociale dell'ICF costituisce un modello culturale e concettuale a forte valenza inclusiva, utile a migliorare la qualità del processo di integrazione poiché orienta verso l'individuazione e l'analisi dei fattori contestuali, degli aspetti inerenti la partecipazione nella comunità di appartenenza, nonché dei facilitatori e delle barriere che, influenzando il processo di integrazione, influenzano la qualità di vita di ciascuna persona.

Applicato in ambito educativo e scolastico il modello indica, codificandolo a livello internazionale, che la rimozione delle barriere che ostacolano l'apprendimento e la partecipazione di tutti gli alunni alla vita scolastica è un dovere primario per l'educazione e la didattica inclusive che si declina anche mediante l'introduzione di un numero crescente di facilitatori.

In questo senso, l'ICF sottolinea l'importanza della capacità di interpretare la realtà scolastica e si propone per un verso, come una risorsa potenzialmente rilevante al fine di individuare analiticamente gli elementi contestuali che condizionano il livello di attività dell'allievo/a e qualificano il suo grado di partecipazione sociale, e per l'altro, come un agente di cambiamento significativo dell'assetto culturale, organizzativo e pedagogico-didattico dei contesti. 


\section{INDAGINI QUANTITATIVE NEGLI STUDI DEI DSA E DELLE DISABILITÀ IN AMBITO NAZIONALE}

In riferimento agli studi sui DSA in contesto italiano, di rilievo è quello condotto dall'Istituto di Ricovero e Cura a Carattere Scientifico (IRCCS) "Burlo Garofalo» ${ }^{18}$ di Trieste nel 2012. Si tratta di uno studio pilota in Friuli Venezia con l'obiettivo di stimare in particolare la reale prevalenza della dislessia sul territorio nazionale, rispetto agli altri disturbi specifici. Sono state coinvolte 94 classi quarte della scuola primaria, e circa 1400 alunni sono stati sottoposti, in momenti successivi, a tre livelli di approfondimento mediante test standardizzati, due dei quali condotti a scuola e uno presso il Centro di Neuropsichiatria Infantile dell'IRCCS stesso.

Dallo studio è emersa la prevalenza della dislessia nel 3,1\% della popolazione considerata e che solo l'1,1\% delle bambine e dei bambini possedeva una diagnosi di dislessia precedente a tale studio. Si può quindi affermare che la dislessia appare essere una condizione frequente ma sottostimata, se si considera che 2 bambini dislessici su 3 non vedono riconosciuto il loro disturbo durante gli anni della scuola primaria, anche quando è chiaramente identificabile.

$\mathrm{Da}$ sottolineare inoltre in questa sede sono le importanti statistiche sull'andamento degli alunni con DSA che risultano iscritti nelle scuole italiane e che vengono pubblicate periodicamente dal Ministero dell'Istruzione dell'Università e della Ricerca, a partire dal mese di febbraio 2011, quando si è svolta la prima rilevazione conseguente all'approvazione della Legge n. 70 del 2010. Nell'ultimo rapporto pubblicato con i dati aggiornati al 15 febbraio 2013, vengono riportati i confronti fra i valori degli iscritti negli anni scolastici 2010/11 e 2011/12, dai quali si rileva un aumento degli alunni con DSA pari a circa il 37\%. Infatti, rispetto al totale di 65.219 (0,9\% della popolazione scolastica) si passa alla cifra di 90.030 (1,2\% della popolazione scolastica).

La percentuale degli alunni con DSA nel sistema nazionale di istruzione supera di poco l' $1 \%$, a fronte di una incidenza media che, secondo le indagini epidemiologiche, si attesterebbe fra il 3\% ed il 5\% dell'intera popolazione. Si tratta evidentemente di una disparità numerica che necessita di

18 L'Istituto Burlo è stato riconosciuto IRCCS il 10 dicembre 1968. Si caratterizza per l'alta specializzazione e per il rilievo nazionale nel settore pediatrico ed in quello della tutela della maternità e della salute della donna. Persegue finalità di ricerca nel campo biomedico ed in quello dell'organizzazione dei servizi sanitari, di innovazione dei modelli d'assistenza e di trasferimento delle conoscenze. Eroga prestazioni di ricovero e cura ad elevata qualificazione a minori, donne in gravidanza, puerpere e donne con patologie ginecologiche, a cittadini italiani e stranieri. L'istituto è sede di attività didattica e di ricerca e di servizi diagnostici universitari, la cui natura e funzionamento sono disciplinati da una specifica convenzione con l'Università degli Studi di Trieste. 
approfondimenti e riflessioni, non rinviabili. Interessante inoltre analizzare i dati suddividendoli secondo i vari gradi di istruzione, come riportato nella Figura 4 (dai quali risulta l'incremento di 10.919 unità $(+39 \%)$ nella scuola secondaria di I grado, a fronte di quello di 5.345 unità $(+24 \%)$ nella scuola primaria, e di $8.547(+54 \%)$ nella scuola secondaria di II grado. Il dato inferiore della scuola primaria può essere spiegato anche dal fatto che, fino al termine della classe seconda, non è possibile rilevare l'esistenza di un DSA.

In riferimento agli studi sulla disabilità in Italia, a partire in particolare dalla Legge-quadro n. 104/1992 sull'assistenza, integrazione sociale e i diritti delle persone con disabilità, è invece possibile rilevare in maniera univoca gli alunni con disabilità che, con apposite certificazioni, frequentano i diversi ordini di scuola. Dalle più recenti statistiche del MIUR, relative all'anno scolastico 2012/13, si evince che il totale degli alunni con disabilità sono risultati complessivamente 222.917 (scuole statali e non statali) pari al 2,5\% dell'intera popolazione scolastica (prossima a 9 milioni di alunni). Rispetto all'anno scolastico precedente tale valore è cresciuto del 3,2\%, registrando tassi di incremento differenti nei vari ordini e gradi di scuola. Si osserva, infatti, un aumento del 2,2\% nella scuola dell'infanzia, del 3,4\% nella scuola primaria, del 2,4\% nella scuola secondaria di I grado e del 4,3\% nella scuola secondaria di II grado (Figura 5).

Con riferimento alla sola scuola statale, dall'anno scolastico 2000/01 gli alunni con disabilità sono aumentati di oltre il $60 \%$ giungendo, nell'anno scolastico 2012/13, ad oltre 205 mila unità. La crescita è stata disomogenea nelle diverse macro aree del territorio nazionale: $+90 \%$ nel Nord-Ovest, $+76 \%$ nel Nord-Est, $+82 \%$ nel Centro, $+34 \%$ nel Mezzogiorno (Figura 6).

Per quanto riguarda l'anno scolastico 2013/14, il MIUR (2013d) ha presentato nel mese di ottobre 2013 alcune anticipazioni rispetto alla numerosità degli alunni con disabilità frequentanti le scuole statali (Figura 7), facendo però ricorso a una fonte di dati differente rispetto a quelle solitamente utilizzate $^{19}$, dalla quale si evince una lieve differenza rispetto al numero degli allievi censiti nell'anno scolastico 2012/13 (205 mila unità nel 2013/14; 202 mila unità nel 2012/13) secondo un trend crescente, già descritto.

19 Per lo studio e la realizzazione dei Focus del Servizio Statistico del MIUR A.S. 2012/13 si riporta il comunicato del MIUR stesso a tale riguardo: «Per l'integrazione scolastica degli alunni con disabilità - Dati statistici» (ottobre 2013) viene utilizzata la seguente Fonte (pag. 4 del Focus): Il Focus elabora i dati sugli alunni con disabilità nell'anno scolastico 2012/2013, rilevati dal Servizio Statistico del MIUR attraverso le Rilevazioni sulle scuole - ex Integrative (con riferimento gli alunni) ed il Fascicolo (con riferimento ai docenti)». Per l'anticipazione dei dati relativi all'A.S. 2013/14, si veda MIUR (novembre 2013): «Anticipazione dati statistici sugli alunni con disabilità e sostegno - A.S. 2013/2014». Per la Fonte di tale Report cfr. p. 1 del documento MIUR, novembre 2013. 


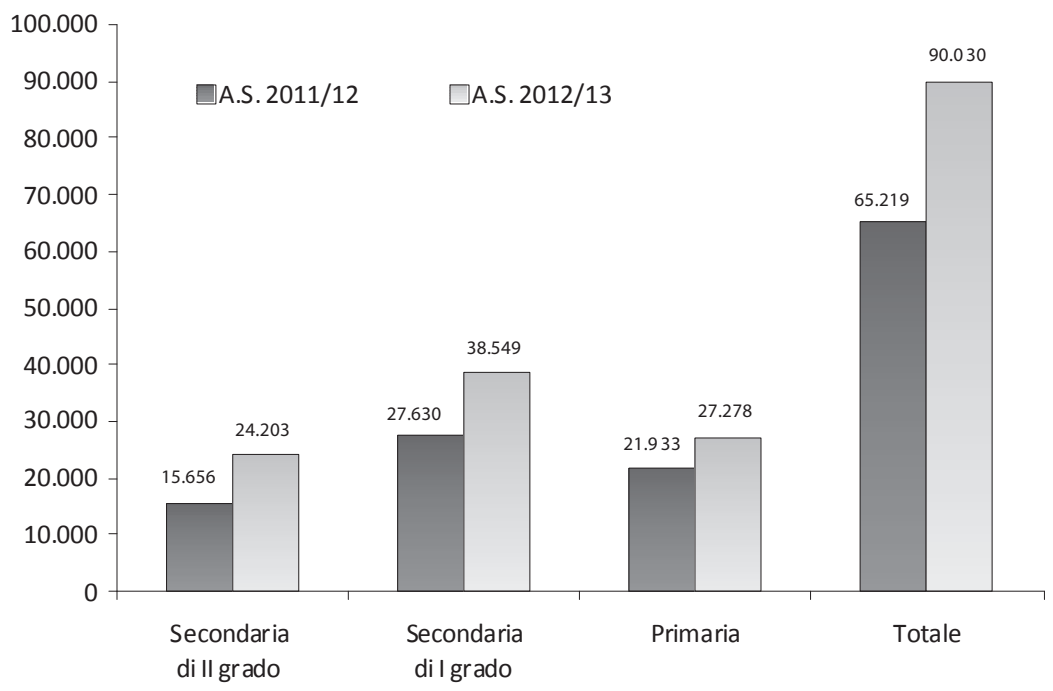

Figura 4. - Alunni con disturbi specifici di apprendimento. A.S. 2010/11-2011/12. Fonte: MIUR - D. G. per gli Studi, la Statistica e i Sistemi Informativi - Servizio Statistico (dati aggiornati al 15 febbraio 2013).

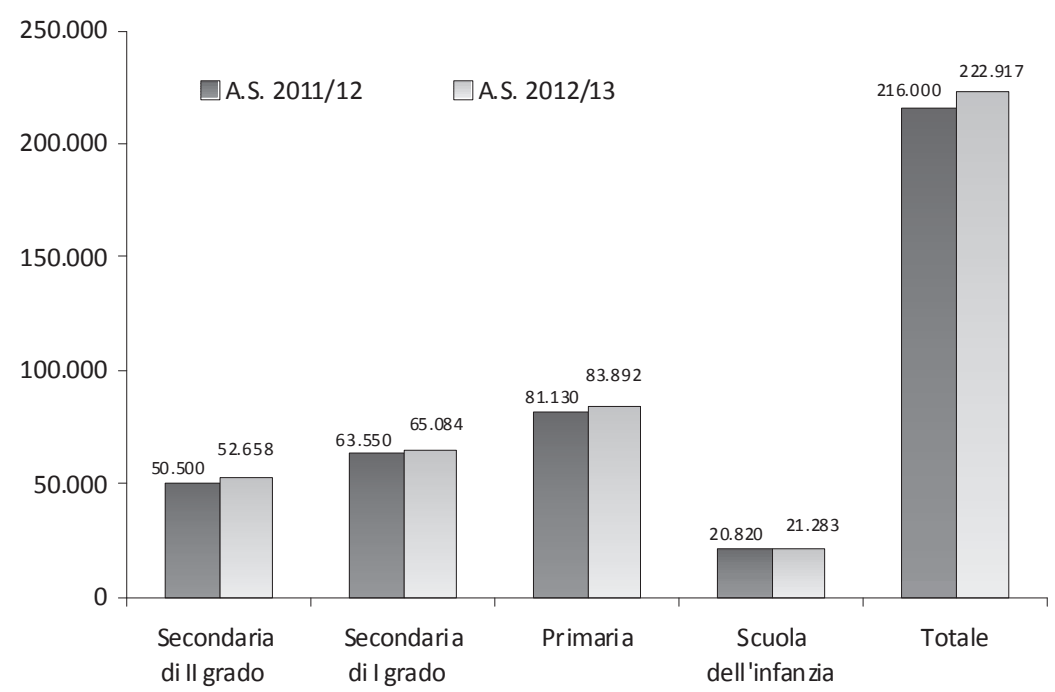

Figura 5. - Alunni con disabilità per ordine e grado. Scuole statali e non statali. AA.SS. 2011/12-2012/13. Fonte: Elaborazione dati MIUR - A.S. 2012/13: "Lintegrazione scolastica degli alunni con disabilità- Dati statistici» (ottobre 2013). 


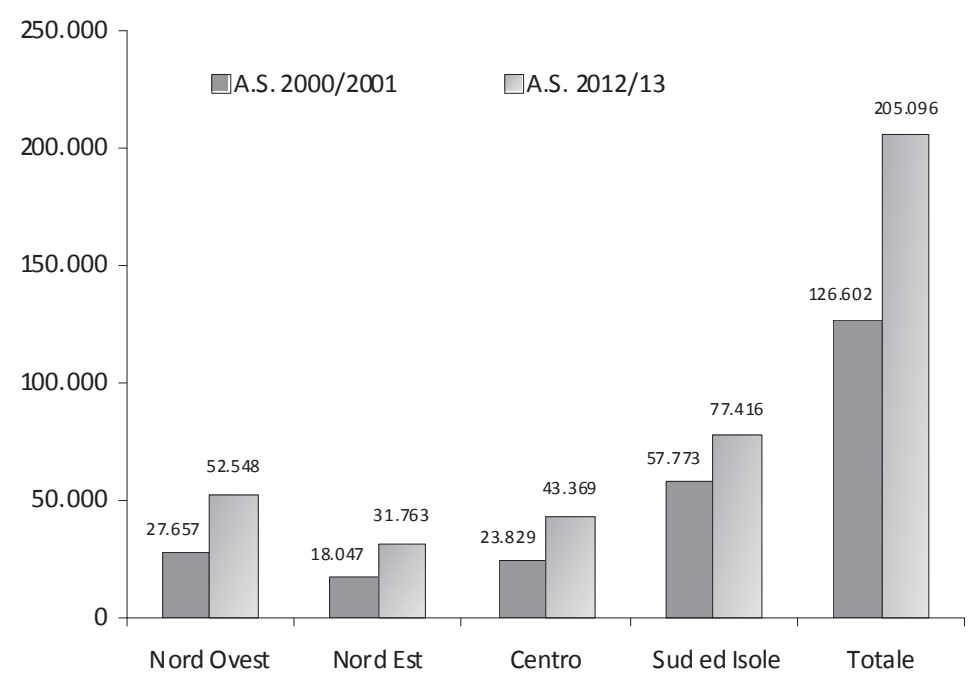

Figura 6. - Alunni con disabilità per area geografica. Scuole statali. AA.SS. 2011/12-2012/13.

Fonte: Elaborazione dati MIUR - A.S. 2012/13:

"L'integrazione scolastica degli alunni con disabilità- Dati statistici» (ottobre 2013).

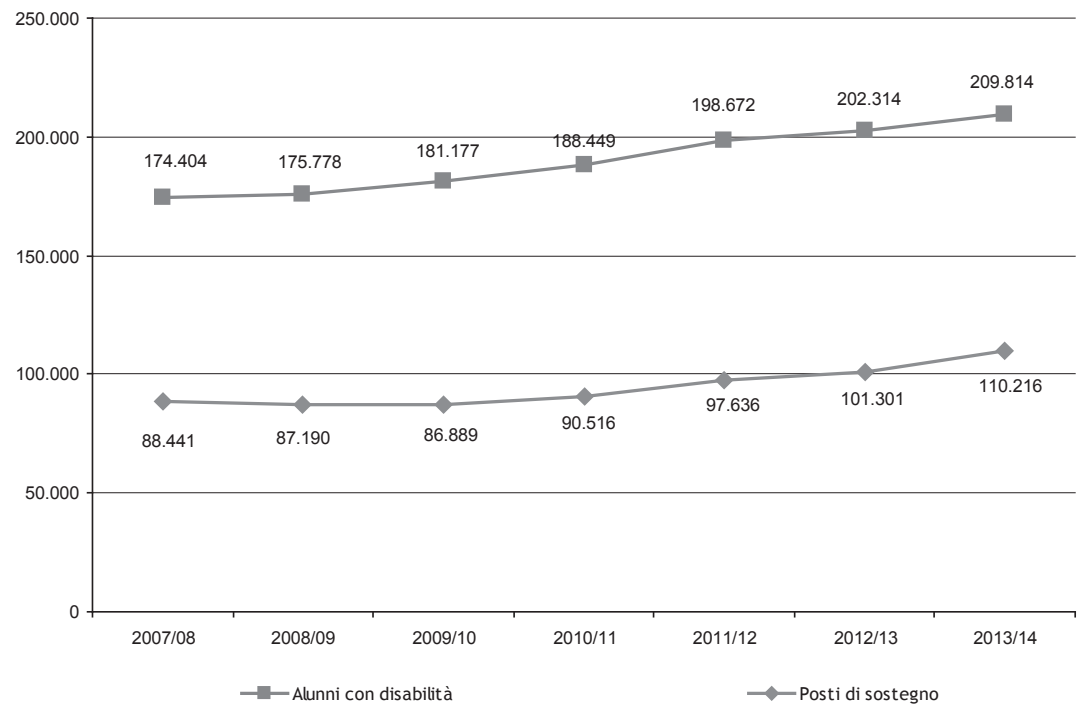

Figura 7. - Alunni con disabilità e posti di sostegno nella scuola statale. AA.SS. 2007/08-2013/14.

Fonte: Elaborazione dati MIUR - A.S. 2013/14:

"Anticipazione dati statistici sugli alunni con disabilità e sostegno» (ottobre 2013). 
Inoltre, nella stessa Figura 7 sono riportati i valori relativi agli insegnanti su posto di sostegno presenti nelle scuole statali dall'anno scolastico 2007/08 al 2013/14: anche per tali valori si evidenzia un trend crescente negli anni considerati. Tale discrepanza numerica, anche se lieve è rappresentativa di uno degli aspetti di cui bisogna tener conto nelle fasi di analisi e confronto dei dati quando si riferiscono, come è stato detto in precedenza, a differenti banche dati progettate per diverse finalità.

Sullo stesso fenomeno, anche nell'ottica di ampliare l'informazione statistica sulla disabilità, in base a quanto richiesto dall'ICF e dalla CRPD, l'ISTAT (Istituto Nazionale di Statistica), con la collaborazione del Ministero del Lavoro e delle Politiche Sociali e del Ministero dell'Istruzione, Università e Ricerca, effettua annualmente una rilevazione sull'inserimento degli alunni con disabilità nelle scuole primarie e secondarie di I grado, statali e non statali, che va a integrare le informazioni già rilevate dal MIUR (ISTAT, 2013). L'indagine si pone l'obiettivo di rilevare le risorse e gli strumenti di cui sono dotati i singoli plessi scolastici per favorire l'inserimento scolastico degli alunni con disabilità, nonché le attività programmate ${ }^{20}$.

A differenza delle statistiche sopra descritte e rilevate dal MIUR che riguardano tutte le scuole italiane di ogni ordine e grado, l'indagine ISTAT è stata a carattere censuario per quanto riguarda le informazioni relative alle scuole primarie e secondarie di primo grado (statali e non statali) quali risorse, attività e strumenti di cui si sono dotati i singoli plessi scolastici per favorire l'inserimento scolastico degli alunni con disabilità; mentre è stata a carattere campionario ${ }^{21}$ per la parte relativa alla numerosità degli alunni con

20 Già attraverso la Legge n. 162/98, l'ordinamento italiano ha riconosciuto l'importanza delle statistiche e della raccolta dati in materia di disabilità rispondendo, pertanto, alla consolidata attività d'indirizzo internazionale e comunitaria in materia. Il nuovo art. 41-bis della Legge n. 104/92 stabilisce che il Ministro per la Solidarietà Sociale promuove indagini statistiche e conoscitive sull'handicap. Su tali basi, l'allora Dipartimento per gli Affari Sociali della Presidenza del Consiglio (oggi Ministero Lavoro e Politiche Sociali) e l'ISTAT hanno avviato sin dal 2000 un'importante collaborazione dalla quale è scaturito il progetto, inizialmente denominato "Sistema informativo sull'handicap", e, in seguito, "Sistema di informazione statistica sulla disabilità». L'obiettivo era quello di creare uno strumento informativo statistico sul tema della disabilità volto, da un lato, a fornire un supporto informativo sia per l'attuazione e il monitoraggio delle politiche sulla disabilità, come previsto dalla Legge n. 104/92 e successive modifiche (Legge n. 162/98), sia per la diffusione ad un pubblico più ampio (cittadini, associazioni, mass media e comunità scientifica nazionale e internazionale) dei dati ufficiali disponibili in Italia sulla disabilità, attraverso il portale www.disabilitaincifre.it. Per garantire alle PcD (Persone con Disabilità) l'accesso e la consultazione dei dati presenti sul suddetto portale, questo è stato costruito in maniera conforme alla normativa sull'accessibilità (art. 9).

21 Il disegno di campionamento è a due stadi di selezione con stratificazione delle unità di primo stadio. Le unità di primo stadio sono le scuole, stratificate per regione geografica e ordine scolastico. Le unità di secondo stadio sono gli alunni con disabilità. La numerosità 
disabilità nelle stesse scuole. Le informazioni sono state rilevate con un questionario elettronico ${ }^{22}$ somministrato via web mediante l'utilizzo del sistema di data capturing INDATA dalle quali si evince un trend crescente degli alunni con disabilità presenti a scuola (Figura 8 ).

Da tale indagine è emerso che nell'anno scolastico 2012/13 gli alunni con disabilità erano circa 149 mila. Di questi, circa 84 mila (pari al 3,0\% del totale) nella scuola primaria e poco più di 65 mila (il 3,7\% del totale) in quella secondaria di primo grado.

Nel primo caso si rileva un aumento di 0,1 punti rispetto all'anno precedente, mentre nel secondo caso l'aumento è di 0,2 punti, confermando la tendenza osservata negli ultimi dieci anni.

Nelle scuole primarie il 21,4\% degli alunni con disabilità non è autonomo in almeno una delle attività indagate (autonomia «nel mangiare, nello spostarsi e nell'andare in bagno», ISTAT, 2013, p. 1), mentre l' $8,0 \%$ non lo è in tutte e tre le attività.

Nelle secondarie di primo grado le percentuali sono rispettivamente del $14,7 \%$ e del $5,5 \%$. Il ritardo mentale, i disturbi del linguaggio, quelli dell'apprendimento e dell'attenzione rappresentano i problemi più frequenti negli alunni con disabilità in entrambi gli ordini scolastici considerati.

Un ulteriore approfondimento sui dati di trend rappresentati da ISTAT e MIUR è stato svolto nel 2011 dall'Associazione Treellle, Caritas Italiana, Fondazione Giovanni Agnelli con il Rapporto Gli alunni con disabilità nella scuola italiana: bilancio e proposte che conclude proponendo «alcune linee progettuali per elaborare un nuovo modello capace di realizzare compiutamente gli straordinari principi e valori che l'Italia, come è riconosciuto anche a livello internazionale, ha fatto propri in materia di integrazione scolastica» (p. 191).

campionaria di primo e di secondo stadio è stata definita tenendo conto sia di esigenze organizzative e di costo, sia degli errori di campionamento attesi delle principali stime di interesse a livello dei domini di stima sopra menzionati. La dimensione complessiva del campione di scuole è stata fissata a 3000 unità.

22 Il questionario elaborato dall'ISTAT, relativo al campione di alunni ha rilevato le seguenti informazioni: Tipologia di disabilità, Diagnosi, Presenza di autonomia, Uso di ausili assistivi e di ausili didattici, Tipo di certificazione, Età, Classe, Ore di sostegno settimanali, Ore settimanali dell'Assistente educativo culturale, Orario di frequenze delle lezioni in classe e fuori classe, Partecipazione alle attività extrascolastiche in città e fuori città. Per approfondimenti si rinvia al Report ISTAT (2013). Anno scolastico 2011/12. L'integrazione degli alunni con disabilità nelle scuole primarie e secondarie di primo grado statali e non statali. 


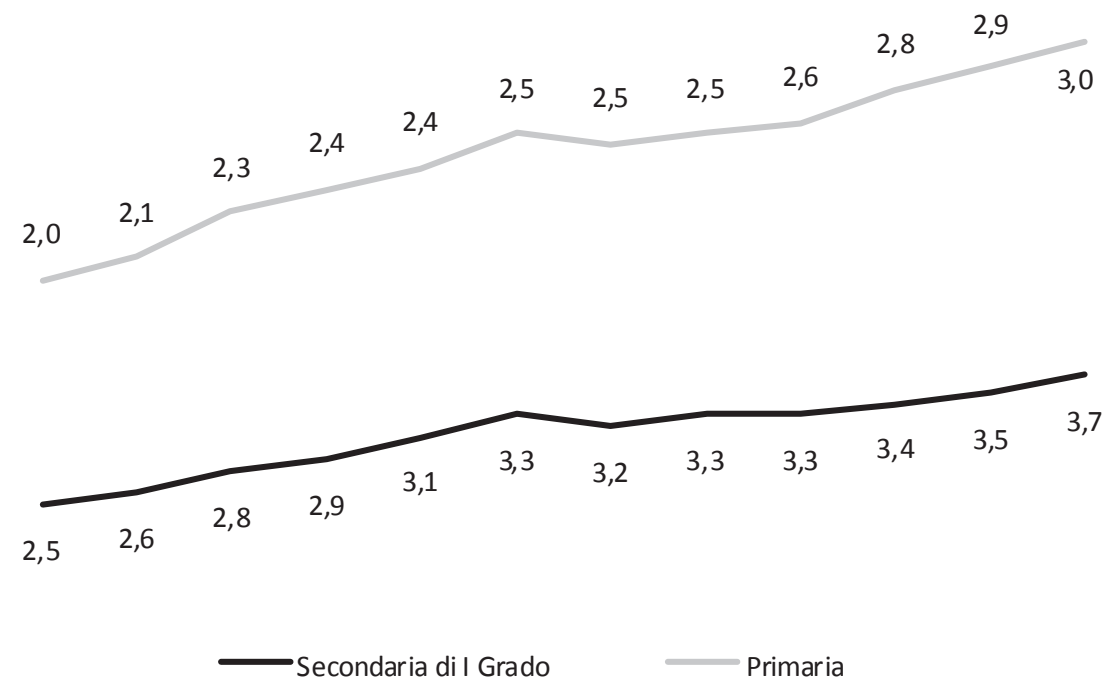

2000/01 2001/02 2003/04 2004/05 2005/06 2006/07 2007/08 $\quad 2008 / 09 \quad 2009 / 10 \quad 2010 / 11 \quad 2011 / 12 \quad 2012 / 13$

Figura 8. - Alunni con disabilità per ordine scolastico e anno scolastico. A.S. 2012/13, valori per 100 alunni con disabilità.

Fonte: Elaborazione ISTAT su dati MIUR.

\section{ASPETTI METODOLOGICI E PROSPETTIVE DI RICERCA}

Come si evince dalla sintesi proposta nel presente lavoro, Organizzazioni internazionali quali in particolare l'UNESCO, la WHO e l'OECD hanno fornito importanti contributi conoscitivi in relazione a problemi di rilevazione e misurazione connessi al trattamento delle informazioni, e hanno evidenziato nello stesso tempo che il pur rilevante sforzo di ciascuna organizzazione non è sufficiente a fornire quadri informativi di organicità, relativi ai DSA e alla disabilità, utili a descrivere dati comparabili e onnicomprensivi.

A partire da tale situazione, per individuare linee di intervento efficaci ai processi di attuazione di programmi inclusivi nei vari Paesi, si rende quindi necessaria una pluralità di azioni che siano condivise nelle modalità di intervento e accomunate da una base di significati e di modelli teorici di riferimento, al fine sia di promuovere la massima corrispondenza tra i diversi 
dispositivi nazionali e la loro messa a sistema, sia di superare la frammentarietà che fatalmente deriva dalla specificità degli interventi e determina la perdita di efficacia, anche a livello locale.

Allo stesso modo, anche in ambito nazionale accade che le statistiche, particolarmente nel campo educativo, necessitino di essere integrate dai dati raccolti con ulteriori ricerche campionarie, come avviene ad esempio nel nostro Paese tra MIUR ed ISTAT, per sviluppare conoscenze sia sulla numerosità degli allievi con disabilità nelle istituzioni scolastiche sia sull'individuazione e sul coordinamento delle loro specifiche esigenze.

Proprio per la connotazione composita ed inclusiva delle diverse istanze, tale approccio integrato costituisce una scelta metodologica di rilevante efficacia anche nell'ottica di un ripensamento del sistema statistico nazionale sulla disabilità avviato nel nostro Paese dall'anno 2011 dall'ISTAT in collaborazione con il Ministero del Lavoro e delle Politiche Sociali e il Ministero dell'Istruzione, Università e Ricerca, attraverso un importante lavoro di inquadramento delle fonti statistiche a partire dai termini e dai nodi concettuali dell'ICF basati sul concetto di disabilità biopsicosociale.

Da questo punto di vista, infatti, se «i funzionamenti» delle persone con disabilità costituiscono il fondamento essenziale di un sistema di valutazione, l'ICF esercita il ruolo di un linguaggio nonché di un integratore semantico per la descrizione anche dell'ambiente rispetto al quale i funzionamenti stessi sono valutati.

È noto, inoltre, che progettare indagini conoscitive implica ovviamente un importante intreccio tra gli aspetti tecnico-metodologici e la concettualizzazione del fenomeno, in tutti i campi e non solo nella ricerca educativa, al fine di poter individuare e puntualizzare tutte quelle fasi che compongono l'intero processo di ricerca: dalla focalizzazione e contestualizzazione del problema, alla formulazione del disegno della ricerca, dalla scelta e messa a punto degli strumenti di rilevazione dati, alla raccolta dati, per giungere alla fase conclusiva di interpretazione, comunicazione e diffusione dei risultati (Lucisano \& Salerni, 2012, p. 47).

Da un punto di vista più generale, è opportuno sottolineare che l'uso dei dati statistici deve essere valorizzato dall'attribuzione di autorevolezza alle fonti delle organizzazioni che costruiscono e diffondono i dati, alle modalità di raccolta e di concettualizzazione dei fenomeni misurati, oltre che alle ulteriori fonti da cui possono provenire statistiche diverse (Domenici, 2006).

A tale riguardo, risulta ancora oggi che, nonostante le informazioni disponibili che possono fornire dati e confronti sistematici ben impiegabili da parte degli operatori scolastici nelle decisioni didattiche più adeguate ai profili degli allievi con DSA e con disabilità, quali quelle provenienti dall'UNESCO, dall'OECD e dalla WHO citate in queste pagine, e quelle 
acquisibili mediante la consultazione di banche dati on line, come quelle sui siti dell'ISTAT e dell'OECD, esse sono in realtà poco conosciute dai ricercatori e studiosi del settore e poco recepite nelle prassi scolastiche. Pertanto accade che gran parte delle informazioni che circolano o nella letteratura di riferimento e/o nella convegnistica, si presentano per lo più con «un taglio spesso aneddotico e con una scarsa attenzione alla valutazione sistematica e comparativa dei risultati» (Calvani, 2012, p. 8).

\subsection{Evidence Based Research e confrontabilità dei dati: quale contributo alle indagini quantitative negli studi sulla disabilità e sui DSA?}

A fronte di tale scenario, il problema di poter disporre di risultati di ricerca che siano omogenei e confrontabili a livello nazionale ed internazionale è stato affrontato anche alla luce dell'innovativa pista di ricerca fondata sulla cultura dell'evidenza e definita con l'espressione Evidence Based Education $\mathrm{EBE}^{23}$ (Calvani, 2012 e 2013).

L'EBE offre un approccio secondo il quale la ricerca è finalizzata in primo luogo a definire standard rigorosi e qualitativamente elevati; in secondo luogo, a rendere fruibili, previe opportune operazioni di comparazione e sintesi, i risultati disponibili in letteratura sull'argomento.

Tali operazioni sono condotte generalmente con l'impiego di tecniche rigorose quali revisioni sistematiche (systematic review) e meta-analisi (metaanalysis) (Hattie, 2009 e 2012) tramite le quali è possibile produrre comparazioni e sintesi metodologicamente fondate dei risultati di ricerca al fine di acquisire un corpus di conoscenze sempre aperto a nuove rielaborazioni e caratterizzato da un livello di generalità e trasferibilità maggiore rispetto alle singole ricerche (Calvani, 2012).

A quest'ultimo riguardo, non trascurabile è l'interpretazione della prospettiva dell'EBE in senso più radicale da parte di alcuni studiosi, i quali tendono ad assumere, come base di conoscenze, principalmente ricerche per esperimento ovvero basate sulla costituzione casuale di gruppi sperimentali e gruppi di controllo e con la somministrazione di uno stimolo sperimentale in ambienti strettamente controllati (Campbell \& Stanley, 1963; Cohen, Manion, \& Morrison, 2007).

Da considerare, inoltre, le situazioni per le quali la logica della comparazione sperimentale su cui si fonda l'EBE, presenta alcune problematiche come nel caso dell'ambito educativo speciale, in quanto si rendono più dif-

23 Il significato di EBE comprende l'analisi dell'efficacia delle pratiche sottostanti i processi di insegnamento-apprendimento. 
ficili indagini sperimentali con gruppi di controllo, a causa dell'ampia variabilità dei problemi che i soggetti presentano e che rende molto difficoltosa se non impossibile la definizione di gruppi omogenei, rendendo necessario ricorrere ad altre metodologie, come disegni centrati su singoli soggetti o metodi misti (Calvani, 2012).

Tali approcci metodologici di tipo misto, in considerazione della ricerca Evidence Based Education che, come detto in precedenza, ritiene accettabili solo indagini di tipo Randomized Controlled Trial (RCT) ${ }^{24}$, possono tuttavia riguardare un'applicazione meno «rigida» delle procedure EBE che può «accogliere anche indagini quasi sperimentali o raccolte empiriche sistematiche oppure osservazioni ripetute in condizioni controllate» (Calvani, 2012, p. 26).

In tale dimensione scientifica, può essere fondata e auspicabile, l'ipotesi di realizzare indagini quantitative anche negli studi sulla disabilità e sui DSA, secondo un approccio flessibile dell'EBE, ma fortemente rigoroso, volto ad orientare gli sforzi della ricerca a favore di una conoscenza affidabile e spendibile nelle politiche e nelle pratiche operative in tali ambiti (Vivanet, 2013) che, come si è delineato nelle pagine precedenti, stenta ad affermarsi in ambito nazionale e internazionale.

Ciò sarebbe promozionale nei confronti della possibilità sia di utilizzo di dati da confrontare in forma sincronica e diacronica rispetto al fenomeno che si sta misurando, sia di realizzazione di «forme di comparazione con altre indagini e capitalizzazione dei risultati» (Calvani, 2012, p. 17) con indiscutibili vantaggi per la comunità educativa e per la didattica inclusiva.

Partendo dall'implicazione epistemologica del concetto di «evidenza» alla luce della quale questa non coincide con quello di verità certa, ma con il risultato di un processo esplicito, riproducibile, falsificabile, reso trasparente, e da sottoporre a gradi probabilistici di affidabilità, l'EBE si caratterizza per la "completa esplicitazione delle proprie assunzioni valoriali o scientifiche, delle metodologie e criteri impiegati» e si attiene "a ben definite procedure, in modo da presentarsi trasparente alla valutazione esterna» (Calvani, 2012, p. 17).

Se utilizzato per rielaborare indagini quantitative sulla disabilità e sui DSA, l'EBE potrebbe quindi contribuire a fornire informazioni, attendibili e valide ai vari decisori, insegnanti, dirigenti scolastici, specialisti e altri operatori, che guardano con grandi aspettative al mondo della ricerca educativa su questi temi, in attesa di indicazioni spendibili e gestibili da parte loro nella quotidianità scolastica.

In questa ottica, il significato di "evidenza» si confronta pragmaticamente con quello di conoscenza fondata di cui i i decisori possono avvalersi

24 Il metodo RCT impiega un gruppo sperimentale ed un gruppo di controllo casuale. La randomizzazione riguarda la scelta casuale degli elementi che vanno a costituire il campione. 
per attivare processi di miglioramento continuo e progressivamente ri-orientabili, o per risolvere via via i problemi che si hanno di fronte.

Ad uso degli insegnanti, l'EBE può dar vita a strumenti in grado di evidenziare loro gli accrescimenti o meno delle conoscenze che vanno acquisendo (e anche quelle degli allievi), coniugando in questo modo gli esiti dei processi di ricerca con il bagaglio delle conoscenze di ciascuno, e con le competenze professionali e la capacità di giudizio, secondo una circolarità virtuosa che procede dalla ricerca alle pratiche didattiche e viceversa.

Interessante notare, a tale riguardo, che anche l'ICF, come l'EBE, costituisce la base per costruire strumenti che possano sviluppare informazioni conoscitive su ciascun allievo, utilizzando un linguaggio standard, universalmente condiviso e comprensibile. Entrambi presentano caratteristiche di sistematicità e una comune origine in ambito sanitario e il successivo rivolgersi a quello educativo. Promuovono inoltre la progettazione scientifica che comporta la replicabilità delle ricerche e delle pratiche, la capitalizzazione dei risultati e il raccordo tra ricerca e pratica didattica. Ma l'analogia più rilevante nell'ambito del presente lavoro è quella che li accomuna nella possibilità di effettuare confronti e analisi fra gli esiti delle ricerche sviluppando forme e modalità di comparazione dei risultati potendosi basare su specifiche procedure di rilevazione di tipo statistico per armonizzare i differenti approcci di rilevazione dei dati sulla disabilità che si origina in ambienti sfavorevoli, e anche per i DSA.

Tenuto conto di tali considerevoli contributi finalizzati anche ad aiutare la ricerca a presentarsi trasparente e a comprendere, sostanzialmente, what work in what circumstances ovvero il mondo dell'Evidence Based Education (Calvani 2012, p. 10), sarebbe dunque auspicabile la prospettiva di approfondire tali aspetti e individuare eventuali modalità con cui l'ICF possa co-agire con l'EBE per l'individuazione dei problemi realmente significativi nelle scuole, non solo per la disabilità e i DSA, e per la ricerca delle migliori evidenze in grado di orientare verso soluzioni ad essi rispondenti. Ciò significa infatti che gli insegnanti che utilizzano consapevolmente i dati provenienti dall'EBE agiscono con modalità coerenti con i dati di ricerca, in un'ottica di superamento del gap tra l'acquisizione delle conoscenze da parte della ricerca e le decisioni operative. A tale approccio di matrice inclusiva, l'EBE offre «l'attuale stato dell'arte della ricerca per ciò che riguarda l'insegnamento efficace con bambini con bisogni educativi speciali - ossia con tutti i bambini» (Mitchell, 2008), rendendo effettivamente possibile la capitalizzazione dei risultati.

Si tratta, a ben vedere, di mettere gli insegnanti nelle condizioni di conoscere e scegliere tra le opzioni didattiche risultate più efficaci, secondo l'approccio definito Evidence Based Practice, e di assumere le decisioni più adeguate per gli allievi con disturbi specifici di apprendimento o con disabilità, tenendo conto ovviamente dell'assoluta singolarità della persona. 
In questo modo gli insegnanti possono cogliere e utilizzare ciò che, in situazioni che risultano essere state indagate e provate (Whitehurst, 2002) ha dato risposte adeguate a determinati bisogni educativi, pur continuando a sottoporre a prove successive di verifica e falsificazione le conoscenze e le pratiche acquisite.

\section{RIFERIMENTI BIBLIOGRAFICI}

Associazione Treelle, Caritas italiana, e Fondazione Giovanni Agnelli (2011). Gli alunni con disabilità nella scuola italiana: bilancio e proposte. Trento: Erickson.

Barbiero, C., Lonciari, I., Montico, M., Monasta, L., Penge, R., Vio, C., Tressoldi, P. E., Ferluga, V., Bigoni, A., Tullio, A., Carrozzi, M., Ronfani, L. (2012). La prevalenza della dislessia in una popolazione scolastica non selezionata nella Regione Friuli Venezia Giulia. IRCCS Burlo Garofolo. ISSUU. Psichiatria dell'Infanzia e dell'Adolescenza, 79(2), Maggio-Agosto, 413-430.

Booth, T., \& Ainscow, M. (2000; revised 2002). Index for inclusion: Developing learning and participation in schools. Bristol: Centre for Studies on Inclusive Education (CSIE).

Booth, T., \& Ainscow, M. (2008). L'Index per l'inclusione (trad. it.). Trento: Erickson.

Bray, M., Adamson, B., \& Mason, M. (2007). Competitive education research: Approach and methods. Hong Kong: Comparative Education, Research Center (CERC) (trad. it., Educazione comparata. Approcci e metodi di ricerca. Milano: Franco Angeli, 2009).

Calvani, A. (2012). Per un'istruzione evidence-based. Analisi teorico-metodologica internazionale sulle didattiche efficaci e inclusive. Trento: Erickson.

Calvani, A. (2013). Evidence based (informed?) education: neopositivismo ingenuo o opportunità epistemologica? Form@re, 2(13), 91-101.

Campbell, D. T., Stanley, J. C. (1963). Experimental and quasi-experimental designs for research on teaching. In N. L. Gage (Ed.), Handbook of research on teaching (pp. 171-246). Washington, DC: American Educational Research Association. Printed by Rand McNally \& Company.

Cannavò, L., \& Frudà, L. (a cura di). (2012). Ricerca sociale. Dal progetto dellindagine alla costruzione degli indici. Roma: Carocci.

Chiappetta Cajola, L. (2004). Il rapporto tra l'insegnante di sostegno con l'insegnante di classe. Studium Educationis, 3, 599-607.

Chiappetta Cajola, L. (2007). L'impiego funzionale degli strumenti di integrazione scolastica: diagnosi funzionale, profilo dinamico funzionale e piano educativo individualizzato. In A. Canevaro (a cura di), Lintegrazione scolastica degli alunni con disabilità. Trentanni di inclusione nella scuola italiana (pp. 221248). Trento: Erickson. 
Chiappetta Cajola, L. (2012). Didattica del gioco e integrazione. Progettare con l'ICF. Roma: Carocci.

Chiappetta Cajola, L., \& Ciraci, A. M. (2013). Didattica inclusiva. Quali competenze per gli insegnanti? Roma: Armando.

Chiaro, M. (2012). Didattica, ricerca e statistica. Strumenti teorico-operativi per la formazione degli insegnanti. Roma: Aracne.

Ciucci, F. (2012). L'intervista nella valutazione e nella ricerca sociale. Parole di chi non ha voce. Milano: Franco Angeli.

Cobalti, A. (2012). «Obiettivi EFA», «Fini del millennio» e «Le statistiche sull'istruzione nel mondo». I Quaderni del Dipartimento di Sociologia e Ricerca Sociale della Facoltà di Sociologia dell'Università di Trento, 59, Febbraio. http://www. unitn.it/dsrs/8701/quaderni-del-dipartimento.

Cohen, L., Manion, L., \& Morrison, K. (2007). Research methods in education. New York - London: Routledge.

Corbetta, P. (2003). La ricerca sociale: metodologia e tecniche, Voll. 1-4. Bologna: Il Mulino.

De Landsheere, G. (1973). Introduzione alla ricerca in educazione (trad. it.). Firenze: La Nuova Italia.

Domenici, G. (2006). Metodologia della ricerca educativa. Roma: Monolite.

Domenici, G. (a cura di). (2009). Valutazione e autovalutazione per la qualificazione dei processi formativi e-learning. Bari: Pensa Multimedia.

Hattie, J. (2009). Visible learning: A synthesis of over 800 meta-analyses relating to achievement. London - New York: Routledge.

Hattie, J. (2012). Visible learning for teachers: Maximizing impact on learning. London - New York: Routledge.

Hattie, J., \& Gan, M. (2011). Instruction based on feedback. In R. E. Mayer \& P. A. Alexander (Eds.), Handbook of research on learning and instruction (pp. 249-271). New York - London: Routledge.

HMSO-Warnock, M. (1978). Report of the Committee of Enquiry into the education of handicapped children and young people. London: Her Majesty's Stationery Office.

ISTAT (2013). Anno scolastico 2012-2013. L'integrazione degli alunni con disabilità nelle scuole primarie e secondarie di primo grado statali e non statali.

Jenkins, E. (2000). Research in science education: Time for a health check? Studies in Science Education, 35, 1-26.

Kish, L. (1987). Statistical design for research. New York: J. Wiley \& Sons.

Lazarsfeld, P. F. (1966). Concept formation and measurement in the behavioral sciences: Some historical observation. In G. J. Di Renzo, Concepts, theory, and explanation in the behavioral sciences (pp. 144-202). New York: Random House. 
Lucisano, P., \& Salerni, A. (2012). Metodologia della ricerca in educazione e formazione. Roma: Carocci.

Lumbelli, L. (1984). Quantità e qualità nella ricerca empirica in pedagogia. In E. Becchi \& B. Vertecchi (a cura di), Manuale critico della sperimentazione e della ricerca educativa (pp. 101-133). Milano: Franco Angeli.

Lumbelli, L. (2006). Costruzione dell'ipotesi ed astrazione nella pedagogia sperimentale. In A. Bondioli, Fare ricerca in pedagogia (pp. 25-60). Milano: Franco Angeli.

McMillan, J. H., \& Schumacher, S. (2013). Research in education. Lavoisier SAS.

Ministero degli Affari Esteri (2013). Piano d'azione sulla disabilità della Cooperazione italiana.

Ministero del Lavoro e delle Politiche Sociali (2013). Programma di azione biennale per la promozione dei diritti e l'integrazione delle persone con disabilità.

Ministero dell'Istruzione, dell'Università e della Ricerca (2007-2009). I CARE. Imparare, Comunicare, Agire in una Rete Educativa.

Ministero dell'Istruzione, dell'Università e della Ricerca (2009). Linee guida per l'integrazione scolastica degli alunni con disabilità.

Ministero dell'Istruzione, dell'Università e della Ricerca (2010-2012). Dal modello ICF dell'OMS alla progettazione per l'inclusione.

Ministero dell'Istruzione, dell'Università e della Ricerca (2011). D.M. del 12/07/2011, n. 5.669: Linee guida per il diritto allo studio degli alunni e degli studenti con $D S A$.

Ministero dell'Istruzione, dell'Università e della Ricerca (2012). D.M. del 27/12/2012: Strumenti intervento per alunni con bisogni educativi speciali e organizzazione territoriale per l'inclusione scolastica.

Ministero dell'Istruzione, dell'Università e della Ricerca (2013a). Circolare Ministeriale del 6/30/2013, n. 8. D.M. del 27/12/2012: Strumenti d'intervento per alunni con bisogni educativi speciali e organizzazione territoriale per l'inclusione scolastica. Indicazioni operative.

Ministero dell'Istruzione, dell'Università e della Ricerca (2013b). Direzione Generale per gli Studi, la Statistica e i Sistemi Informativi - Direzione Generale per lo Studente, l'Integrazione, la Partecipazione e la Comunicazione, Alunni con disturbi specifici di apprendimento. AA.SS. 2010/2011 e 2011/12.

Ministero dell'Istruzione, dell'Università e della Ricerca (2013c). L'integrazione scolastica degli alunni con disabilità - Dati statistici. A.S. 2012/13.

Ministero dell'Istruzione, dell'Università e della Ricerca (2013d). Anticipazione dati statistici sugli alunni con disabilità e sostegno. A.S. 2013/14.

Ministero dell'Istruzione, dell'Università e della Ricerca (2013e). Bisogni Educativi Speciali. Approfondimenti in ordine alla redazione del piano annuale per l'inclusività nell'ottica della personalizzazione dell'apprendimento. Materiali per la formazione. A.S. 2013/14. 
Ministero dell'Istruzione, dell'Università e della Ricerca e Ministero della Salute (2012). Protocollo d'intesa per la tutela del diritto alla salute e del diritto allo studio degli alunni e degli studenti con disabilità.

Mitchell, D. (2008). What really works in special and inclusive education. London: Routledge.

Newbold, P., \& Carlson, W. L. (2010). Statistica. Milano: Pearson Paravia.

OECD (2005). P. Evans, Statistics and data collection statistics Meeting for South Eastern Europe. Paris, 5 December.

OECD (2012a). Education at a glance. http://www.oecd.org.

OECD (2012b). Social Policy Division - Directorate of Employment, Labour and Social Affairs, in Child well-being Module www.oecd.org/els/social/family/ database/CWBM, May. CX3.1. Special Educational Needs (SEN).

ONU (2006). Convenzione delle Nazioni Unite sui diritti delle persone con disabilità.

Ostillio, M. C. (2005). Customer database: conoscere il cliente per gestire le relazioni. Milano: Egea.

Sen, A. (1992). La diseguaglianza. Bologna: Il Mulino.

Tomblin, J. B., Records, N. L., Buckwalter, P., Zhang, X., Smith, E., \& O’Brien, M. (1997). Prevalence of specific language impairment in kindergarten children. Journal of Speech and Hearing Research, 40, 1245-1260.

Trinchero, R. (2002). Manuale di ricerca educativa. Milano: Franco Angeli.

UNESCO - UIS (1997). International Standard Classification of Education, ISCED 1997 (2nd ed.). UNESCO - Institute for Statistics, 2006. Montreal, Canada. http://www.uis.unesco.org.

UNESCO (2009). Education for all Global Monitoring Report 2009. http://www. unesco.org.

UNESCO-UIS (2012). International Standard Classification of Education, ISCED 2011. UNESCO - Institute for Statistics. Montreal, Canada. http://www.uis. unesco.org.

UNESCO (2012). Education for all Global Monitoring Report 2012. http://www. unesco.org.

UNICEF (2013). La condizione dell'infanzia nel mondo 2013. Bambini e disabilità. http://www.unicef.it/pubblicazioni.

UNIONE EUROPEA - HORIZON (2020). http://ec.europa.eu/programmes/ horizon 2020.

World Health Organization (2001). International Classification of Functioning, Disability and Health (ICF). http://www.who.int.

World Health Organization (2007). International Classification of Functioning, Disability and Health. Children \& Youth (ICF-CY). http://www.who.int.

World Health Organization (2011). World Report on Disability. http://www.who.int. Zammuner, V. L. (2000). Tecniche dell'intervista e del questionario. Bologna: Il Mulino. 
Siti di riferimento (verificati il 31/01/2014)

http://www.undp.org

http://en.unesco.org

http://www.uis.unesco.org

http://www.un.org

http://www.oecd.org

http://www.unicef.it

http://www.who.int

\section{RiassunTO}

Raccogliere, organizzare, descrivere e interpretare dati, rappresentano le fasi che caratterizzano in particolare il processo delle indagini quantitative. Ad esse viene riconosciuta una crescente importanza in una società dove è fondamentale comprendere pienamente le grandi e complesse trasformazioni in atto, in modo da poter rispondere efficacemente alla sfida del miglioramento della qualità dell'struzione per tutti gli allievi. A partire da tale presupposto, questo contributo intende offrire un quadro relativo alle indagini quantitative condotte, sia in ambito nazionale che internazionale, negli studi delle disabilità e dei disturbi specifici di apprendimento, mettendo in luce le criticità connesse alla pluralità degli strumenti e dei metodi di rilevazione di dati e di informazioni impiegati nei diversi Paesi, che non consentono, di fatto, di apportare significati statisticamente comparabili e utili a descrivere i problemi che ostacolano l'apprendimento e la partecipazione degli allievi alla vita scolastica, e a fornire linee di tendenza sul loro andamento. In questa sede si sostiene che limpegno di rappresentare sul piano empirico e operativo una realtà cosi complessa comporta la necessità sia di potenziare lo sforzo di integrazione di competenze sia di poter contare sul rinnovato e decisivo supporto e coinvolgimento di autorevoli organismi, nazionali e internazionali, in grado di ri-orientare metodologicamente le procedure di indagine, superando innanzitutto le criticità legate sia all'uso di concetti, definizioni e classificazioni, sia ai criteri di raccolta dei dati e di gestione dei relativi data-base, tenendo anche in conto la molteplicità degli attori coinvolti, nonché i vincoli di natura legislativa e normativa connessi.

Parole chiave: Classificazione internazionale del funzionamento, Disabilità, Disturbi specifici di apprendimento, Evidence based education, Indagini quantitative. 